\title{
Hybrid Recurrent Laguerre-Orthogonal-Polynomial NN Control System Applied in V-Belt Continuously Variable Transmission System Using Particle Swarm Optimization
}

\author{
Chih-Hong Lin \\ Department of Electrical Engineering, National United University, No. 1, Lienda, Kung-Jing Li, Miaoli City, \\ Miaoli County 36003, Taiwan
}

Correspondence should be addressed to Chih-Hong Lin; jhlin@nuu.edu.tw

Received 18 May 2014; Accepted 1 September 2014

Academic Editor: Kang Li

Copyright (C) 2015 Chih-Hong Lin. This is an open access article distributed under the Creative Commons Attribution License, which permits unrestricted use, distribution, and reproduction in any medium, provided the original work is properly cited.

\begin{abstract}
Because the V-belt continuously variable transmission (CVT) system driven by permanent magnet synchronous motor (PMSM) has much unknown nonlinear and time-varying characteristics, the better control performance design for the linear control design is a time consuming procedure. In order to overcome difficulties for design of the linear controllers, the hybrid recurrent Laguerre-orthogonal-polynomial neural network (NN) control system which has online learning ability to respond to the system's nonlinear and time-varying behaviors is proposed to control PMSM servo-driven V-belt CVT system under the occurrence of the lumped nonlinear load disturbances. The hybrid recurrent Laguerre-orthogonal-polynomial NN control system consists of an inspector control, a recurrent Laguerre-orthogonal-polynomial NN control with adaptive law, and a recouped control with estimated law. Moreover, the adaptive law of online parameters in the recurrent Laguerre-orthogonal-polynomial NN is derived using the Lyapunov stability theorem. Furthermore, the optimal learning rate of the parameters by means of modified particle swarm optimization (PSO) is proposed to achieve fast convergence. Finally, to show the effectiveness of the proposed control scheme, comparative studies are demonstrated by experimental results.
\end{abstract}

\section{Introduction}

A V-belt continuously variable transmission (CVT) [1-8] is typically composed of two hydraulically, or spring, actuated variable radii pulleys and a chain, or metal pushing, belt. To launch a vehicle from rest, the input pulley radius will be smaller than the output pulley radius, resulting in a speed reduction and torque multiplication transmitted to the drive shaft. For increased output shaft speed, the pulley radii are inversely manipulated simultaneously (i.e., input pulley radius increases as the output pulley radius decreases) to maintain constant belt length. A CVT may operate at a specific speed while changing the pulleys' radii to achieve torque multiplication, acceleration, and speed as per the vehicle's velocity, load requirements, engine power, and gear ratios. This operating profile provides the research motivation for CVT dynamics and nonlinear control algorithms. CVT-based vehicles have been traditionally regulated using a standard proportional integral derivative- (PID-) based controller with measurements of the gear ratio [3]. It has also been demonstrated that this control strategy provides satisfactory performance using gain-scheduling with a large set of points. In addition, numerous fuzzy logic controllers [4] have also been proposed. However, V-belt continuously variable transmission (CVT) system driven by alternating current (AC) motor is yet not shown in any commercial reports so that it provides the research motivation in this study.

The AC motor has several types such as permanent magnet synchronous motor (PMSM), switched reluctance motor (SRM), and induction motor (IM). In order to select the appropriate AC motor for driven V-belt CVT system, high efficiency is one of the most important factories to be selected. The PMSM provides higher efficiency, higher power density, and lower power loss for their size compared to SRM and IM. In addition, field-oriented control is one the most popular control techniques for the PMSM servo-driven system. As a result, torque ripple of the PMSM is lower than the SRM 
and IM. On the other hand, the PMSM controlled by fieldoriented control, which can be achieved fast by four-quadrant operation, is much less sensitive to the parameters variation of the motor [9-11]. Therefore, the PMSM has been widely used in many industrial applications such as robotics, electric power steering, and other mechatronics [9-11].

Artificial neural networks (ANNs) have emerged as a powerful learning technique to perform complex tasks such as highly nonlinear approximations and the control of dynamical systems [12-16]. Some of the prime advantages of using $\mathrm{NN}$ are their ability to learn based on optimization of an appropriate error function and their excellent performance for approximation of nonlinear functions. There are different paradigms of NNs proposed by different researchers for the tasks of system identifications and controls [13-16]. One of the major drawbacks of the $\mathrm{NN}$ is that it is computationally intensive and needs large number of iterations for its training. In order to reduce the computational complexity, a functional-link NN, which has shown that it is capable of producing similar performance as that of $\mathrm{NN}$ but with much less computational cost, was reported in [17-19]. These functional-link NNs [17-19] with faster convergence and lesser computational complexity were executed in the identification and controls of nonlinear dynamic system with satisfactory results. Recently, the Laguerre-functional-expansions combined with $\mathrm{NN}$, which was applied in highly nonlinear approximations and the control of dynamical systems, have been proposed [20-24]. Aadaleesan et al. [20] proposed the Laguerre filter combined with the wavelet network in order to approximate the memoryless nonlinearity. Approximation the linear and nonlinear parts of a Wiener structure by means of the Laguerre filter and the general feed-forward NN was reported in [21]. The Laguerre-functional-expansions feed-forward NN, which employed Laguerre-orthogonalpolynomials in the activation functions of the hidden neurons in order to identify models of the chaotic time series, was proposed by Zou and Xiao [22]. Patra et al. [23] proposed a computationally efficient Laguerre $\mathrm{NN}$, which is based on Laguerre-functional-expansions to autocompensate for the associated nonlinearity and environmental dependence for intelligent sensors, and provide linearized sensor readout even when the motes are operated in harsh environments. Patra et al. [24] present an intelligent technique by means of novel computationally efficient Laguerre NN to compensate for the inherent sensor nonlinearity and the environmental influences. Since the Laguerre NN is a single-layer NN, its computational complexity is found to be much lower than a multilayer perception (MLP). However, these Laguerrefunctional-expansions feed-forward NNs without a feedback loops can be used for static function approximation, but they cannot adequately approximate dynamic behaviors found in PMSM servo-driven V-belt CVT system with nonlinear and time-varying characteristics.

The recurrent NN has received increasing attention due to its structural advantage in the modelling of the nonlinear system and dynamic control of the system [25-29]. These networks are capable of effective identification and control of complex process dynamics, but with the expense of large computational complexity. Hence, if each neuron in the recurrent $\mathrm{NN}$ is considered as a state in the nonlinear dynamic systems, the self-connection feedback type is able to approximate the dynamic systems efficiently [25-29]. In order to improve the ability of identifying high order systems and reduce computational complexity, the recurrent Laguerre-orthogonal-polynomial NN, which has more advantages than the Laguerre-orthogonal-polynomial NN including better performance, higher accuracy, and dynamic robustness, has been proposed to control the PMSM servodriven V-belt CVT system with nonlinear and time-varying characteristics in this paper.

Particular swarm optimization (PSO) is a populationbased, self-adaptive search optimization technique first introduced by Kennedy and Eberhart [30]. Similar to genetic algorithms [31], an evolutionary algorithm approach, the PSO is an evolutionary optimization tool of swarm intelligence field based on a swarm (population), where each member is seen as a particle, and each particle is a potential solution to the problem under analysis. The motivation for the development of this method was based on the simulation of simplified animal social behaviors such as fish schooling, bird flocking, and so forth. However, unlike in other evolutionary optimization methods, in PSO there is no direct recombination of genetic material between individuals during the search. The PSO algorithm works on the social behavior of particles in the swarm. Therefore, it finds the global best solution by simply adjusting the trajectory of each individual toward its own best location and toward the best particle of the entire swarm at each time step (generation) $[30,32]$. Clerc and Kennedy [32] introduced the concept of inertia weight to the original version of $\mathrm{PSO}$, in order to balance the local and global search during the optimization process. Thus, PSO has been widely applied in mathematical modeling, dynamic programming, and system control [3336] due to simple structure, simple parameter setting, and fast convergence speed. How to improve the convergence speed and how to guarantee the convergence of PSO are the main problems of PSO improvement [37] and are gradually turning into a hot topic in this field. In order to weigh the relationship between local search and global search, Clerc and Kennedy [32] and Eberhart and Shi [38, 39] proposed improved particle swarm optimization with inertial weight to control the exploitation and exploration. Meanwhile, some researchers [40-42] have proposed the topical improved particle swarm algorithm with inertia factor, which is called topical particle swarm optimization. However, the PSO existed in premature convergence problem and the modified PSO is proposed to prevent premature convergence and to acquire optimal learning rate with better convergence in this paper.

In this study the hybrid recurrent Laguerre-orthogonalpolynomial NN control system is developed to control the V-belt CVT system with many nonlinear dynamics [1-8, 43-46], which is driven by PMSM. The hybrid recurrent Laguerre-orthogonal-polynomial NN control system has fast learning property and good generalization capability. The control method, which is not dependent upon the predetermined characteristics of the motor, can adapt to any change in the motor characteristics. The hybrid recurrent 
Laguerre-orthogonal-polynomial NN control system, which is composed of the inspector control, the recurrent Laguerreorthogonal-polynomial NN control with adaptive law, and the recouped control, is applied to the V-belt CVT system driven by PMSM. The adaptive law of the online parameter in the recurrent Laguerre-orthogonal-polynomial NN can be derived according to the Lyapunov stability theorem and the gradient descent method. The recurrent Laguerreorthogonal-polynomial NN has the online learning ability to respond to the system's nonlinear and time-varying behaviors under the occurrence of the lumped nonlinear external disturbances with parameters variation. Furthermore, two optimal learning rates of the parameters by means of modified PSO are proposed to achieve fast convergence. Finally, the control performances of the proposed hybrid recurrent Laguerre-orthogonal-polynomial NN control system are verified by experimental results.

The paper is structured as follows: Section 2 provides the configuration of the V-belt CVT system driven by PMSM. Section 3 develops the proposed novel hybrid recurrent Laguerre-orthogonal-polynomial $\mathrm{NN}$ control system for controlling the V-belt CVT system driven by PMSM. Section 4 presents the experimental results for comparisons between the proposed control method and PI control method at three cases. Section 5 provides the conclusions.

\section{Configuration of System}

Since the electric scooter system has much unknown nonlinear uncertainties and parameter variations, such as load torque, rolling resistance, wind resistance, and braking force, the V-belt CVT and clutch in the scooter model can be categorized as functioning in one of two operating modes depending on the speed of the V-belt CVT output axis: disengaged or completely coupled. At the start of the PMSM drive cycles, the scooter is in an idle state. The clutch is initially disengaged, and subsequent transition between modes is controlled by the clutch axis rotational speed. Except for the mechanical losses, the PMSM power is transmitted through the V-belt CVT and clutch to the wheel in the electric scooter.

2.1. Structure of the V-Belt CVT System Driven by PMSM. The development of the V-belt CVT began with rubber V-belts [5]. Despite the fact that rubber V-belt CVTs are not well suited for automotive applications because of their limited torque capacity, there are some interesting concepts on the market. The V-belt CVT consists of a segmented rubber Vbelt and two shafts with conical pulleys. The V-belt is clamped between two pairs of conical sheaves. In the V-belt CVT, the transmission ratio is determined by simultaneous adjustment of the running radii of the belt on the pulleys. On each shaft, there is one fixed and one axially moveable sheave. Axial movement of the moveable sheave adjusts the gap between the sheaves and thereby the belt running radius. The input shaft of the V-belt CVT is called the primary shaft which mounted the PMSM, and the output shaft is the secondary shaft which mounted the wheel. The structure of the V-belt
CVT is shown in Figure 1. The appearance of the primary pulley side and the secondary pulley side in the V-belt CVT is shown in Figure 1(a), and the cross-section view of the secondary shaft in the V-belt CVT is shown in Figure 1(b). The wheel of electric scooter is connected to the output shaft of the final reduction using a torsion spring, which models the combined stiffness of both the drive shafts. The electric scooter inertia is connected to the wheel using linear damper, which models the tire force. The rolling resistance on the electric scooter is modeled as a load torque. In order to reduce system complexity, the torque dynamic equations in the primary drive shaft and the secondary drive shaft of the V-belt CVT shown in Figure 2 can be simplified as [1-8]

$$
\begin{gathered}
J_{p} \dot{\omega}_{r}+B_{p} \omega_{r}+T_{p}=T_{e}, \\
T_{s}=J_{s} \dot{\omega}_{s}+B_{s} \omega_{s}+T_{l}^{s}\left(F_{l}\left(B_{g}\right), v_{a}\left(v_{r}, B_{g}\right), \tau_{a}\left(v_{r}\right), \omega_{s}^{2}\right),
\end{gathered}
$$

in which $T_{l}^{s}\left(F_{l}\left(B_{g}\right), v_{a}\left(v_{r}, B_{g}\right), \tau_{a}\left(v_{r}\right), \omega_{s}^{2}\right)[1-8]$ is the lumped nonlinear external disturbances of the secondary drive side on the wheel; $T_{p}=\sigma_{s} \omega_{s} T_{s} / \omega_{r}$ is the drive torque of the primary pulley shaft; $T_{s}$ is the drive torque of the secondary pulley shaft; $\sigma_{s}$ is the conversion ratio with respect to secondary pulley shaft transferred to primary pulley shaft of V-belt arc length; $v_{a}\left(v_{r}, B_{q}\right)$ is the rolling resistance; $\tau_{a}\left(v_{r}\right)$ is the wind resistance; $F_{l}\left(B_{g}\right)$ is a braking force; $v_{r}$ is the total wind velocity; $B_{g}$ represents the total frictional coefficient of ground surface; $B_{p}$ and $B_{s}$ represent the viscous frictional coefficients of the PMSM and the wheel, respectively; $J_{p}$ and $J_{s}$ are the inertias of the PMSM and the wheel, respectively; $\omega_{r}$ and $\omega_{s}$ are the speeds of the PMSM and the wheel, respectively. Then using speed ratio and sliding ratio [1-8], the torque equation can be transformed from the secondary pulley side to the primary pulley side. Therefore, the resultant dynamic equation of the PMSM driven V-belt CVT system from (1) can be simplified as [1-8, 41-45]

$$
\begin{aligned}
J_{r} \dot{\omega}_{r} & +B_{r} \omega_{r}+T_{l}\left(T_{a}, \Delta T_{p}, F_{l}\left(B_{g}\right), v_{a}\left(v_{r}, B_{g}\right), \tau_{a}\left(v_{r}\right), \omega_{r}^{2}\right) \\
& =T_{e},
\end{aligned}
$$

in which $T_{l}\left(T_{a}, \Delta T_{p}, F_{l}\left(B_{g}\right), v_{a}\left(v_{r}, B_{g}\right), \tau_{a}\left(v_{r}\right), \omega_{r}^{2}\right)=T_{a}+$ $\Delta T_{p}+T_{\text {un }}[1-8]$ is the resultant lumped nonlinear external disturbances with parameter variations; $T_{a}$ is the fixed load torque; $\Delta T_{p}=\Delta J_{r} \dot{\omega}_{r}+\Delta B_{r} \omega_{r}$ is the resultant parameter variation; $v_{a}\left(v_{r}, B_{g}\right)$ is the resultant rolling resistance; $\tau_{a}\left(v_{r}\right)$ is the resultant wind resistance; $F_{l}\left(B_{g}\right)$ is the resultant braking force; $T_{\text {un }}=F_{l}\left(B_{g}\right)+v_{a}\left(v_{r}, B_{g}\right)+\tau_{a}\left(v_{r}\right) \omega_{r}^{2}$ is the resultant unknown nonlinear load torque; $B_{r}$ is the resultant viscous frictional coefficient; $J_{r}$ is the resultant moment of inertia.

2.2. Structure of the PMSM Driven System. For convenient design, the stator voltage equations of the PMSM driven 


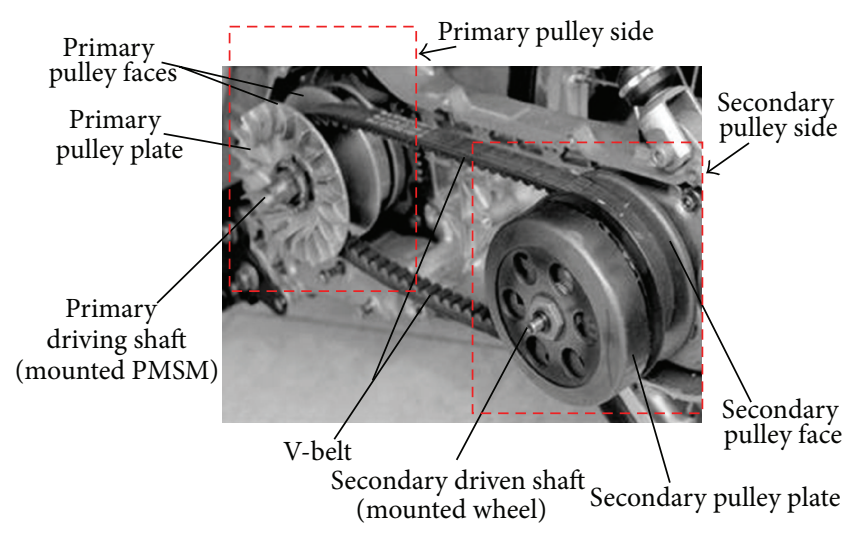

(a)

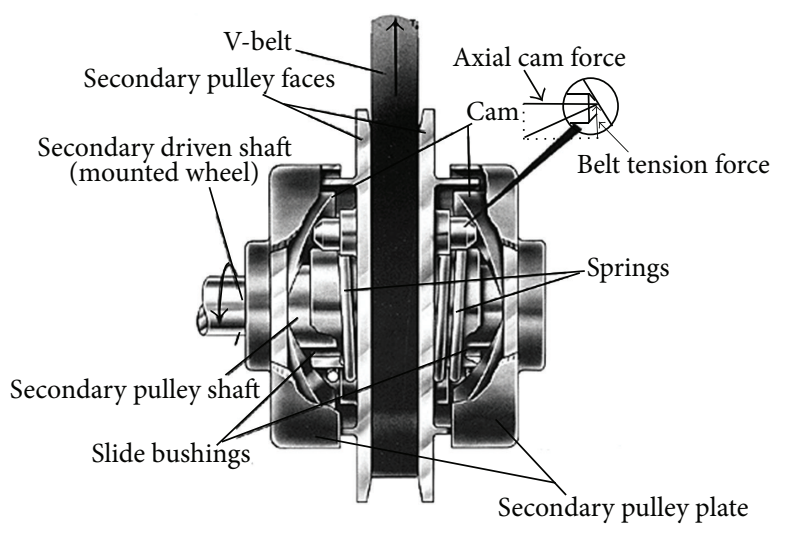

(b)

FIGURE 1: Structure of the V-belt CVT system: (a) appearance of the primary pulley side and the secondary pulley side; (b) cross-section view of the secondary shaft.

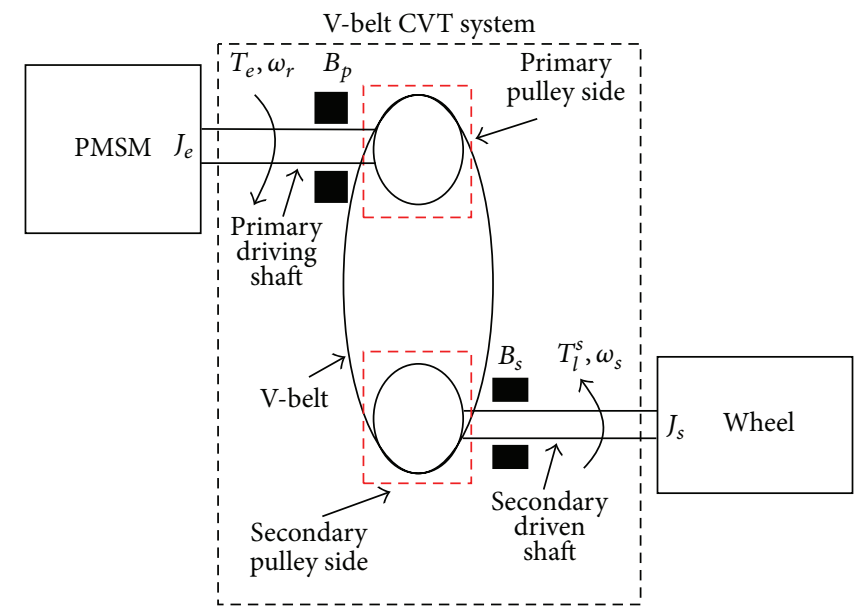

FIGURE 2: Schematic of the PMSM-wheel connection through the V-belt CVT system.

V-belt CVT system in the synchronously rotating reference frame can be described as follows [9-11, 42-45]:

$$
\begin{aligned}
& v_{q r}=R_{r} i_{q r}+L_{q r} \dot{i}_{q r}+\omega_{f}\left(L_{d r} i_{d r}+\lambda_{f d}\right), \\
& v_{d r}=R_{r} i_{d r}+L_{d r} \dot{i}_{d r}-\omega_{f} L_{q r} i_{q r},
\end{aligned}
$$

in which $v_{q r}$ and $v_{d r}$ are the $d$-axis and $q$-axis stator voltages; $i_{q r}$ and $i_{d r}$ are the $d$-axis and $q$-axis stator currents; $L_{q r}$ and $L_{d r}$ are the $d$-axis and $q$-axis stator inductances; $\lambda_{f d}$ is the $d$-axis permanent magnet flux linkage; $R_{r}$ is the stator resistance; $\omega_{f}=P_{r} \omega_{r} / 2$ is the electrical angular speed.

The electromagnetic torque $T_{e}$ of the PMSM driven V-belt CVT system can be described as

$$
T_{e}=\frac{3 P_{r}\left[\lambda_{f d} i_{q r}+\left(L_{d r}-L_{q r}\right) i_{d r} i_{q r}\right]}{4}
$$

in which $P_{r}$ is the number of poles. Due to $L_{d r}=L_{q r}$ for a surface-mounted PMSM, the second term of (4) is zero.
Moreover, $\lambda_{f d}$ is a constant for surface-mounted PMSM. The rotor flux is produced in the $d$-axis only, while the current vector is generated in the $q$-axis for the field-oriented control. When the $d$-axis rotor flux is a constant and torque angle is $\pi / 2$ [9-11], the maximum torque per ampere can be reached for the field-oriented control. The electromagnetic torque $T_{e}$ is linearly proportional to the $q$-axis current $i_{q r}$, which is determined by closed-loop control. The control principle of the PMSM driven system is based on field orientation. The PMSM driven V-belt CVT system with the implementation of field-oriented control can be reduced as $T_{e}=k_{r} i_{q r}$, in which $k_{r}=3 P_{r} \lambda_{f d} / 4$ is the torque constant. The block diagram of the V-belt CVT system driven by PMSM is shown in Figure 3.

The whole system of the PMSM driven V-belt CVT system can be indicated as follows: a field-oriented institution, a current PI control loop, a sinusoidal PWM control circuit, an interlock circuit and an isolated circuit, an IGBT power module inverter, and a speed control loop. The PI current loop controller is the current loop tracking controller. In order to attain good dynamic response, all gains for wellknown PI current loop controller are listed as follows: $k_{\mathrm{pc}}=$ 9.5 and $k_{\mathrm{ic}}=k_{\mathrm{pc}} / T_{\mathrm{ic}}=2.8$ through some heuristic knowledge [47-50] on the tuning of the PI controller. The field-oriented institution consists of the coordinate transformation, $\sin \theta_{f} / \cos \theta_{f}$ generation, and lookup table generation. The TMS320C32 DSP control system manufactured by Spinel Technology Corporation is used to implement fieldoriented control and speed control. The V-belt CVT system driven by PMSM is manipulated under the lumped external disturbances with nonlinear uncertainties.

\section{Design of Hybrid Recurrent Laguerre-Orthogonal-Polynomial NN Control System}

Due tononlinear uncertainties of the V-belt CVT system driven by PMSM such as nonlinear friction force of the transmission belt and clutch, rolling resistance, wind resistance, and braking force, these will lead to degeneration of tracking responses in command current and speed of the V-belt CVT 


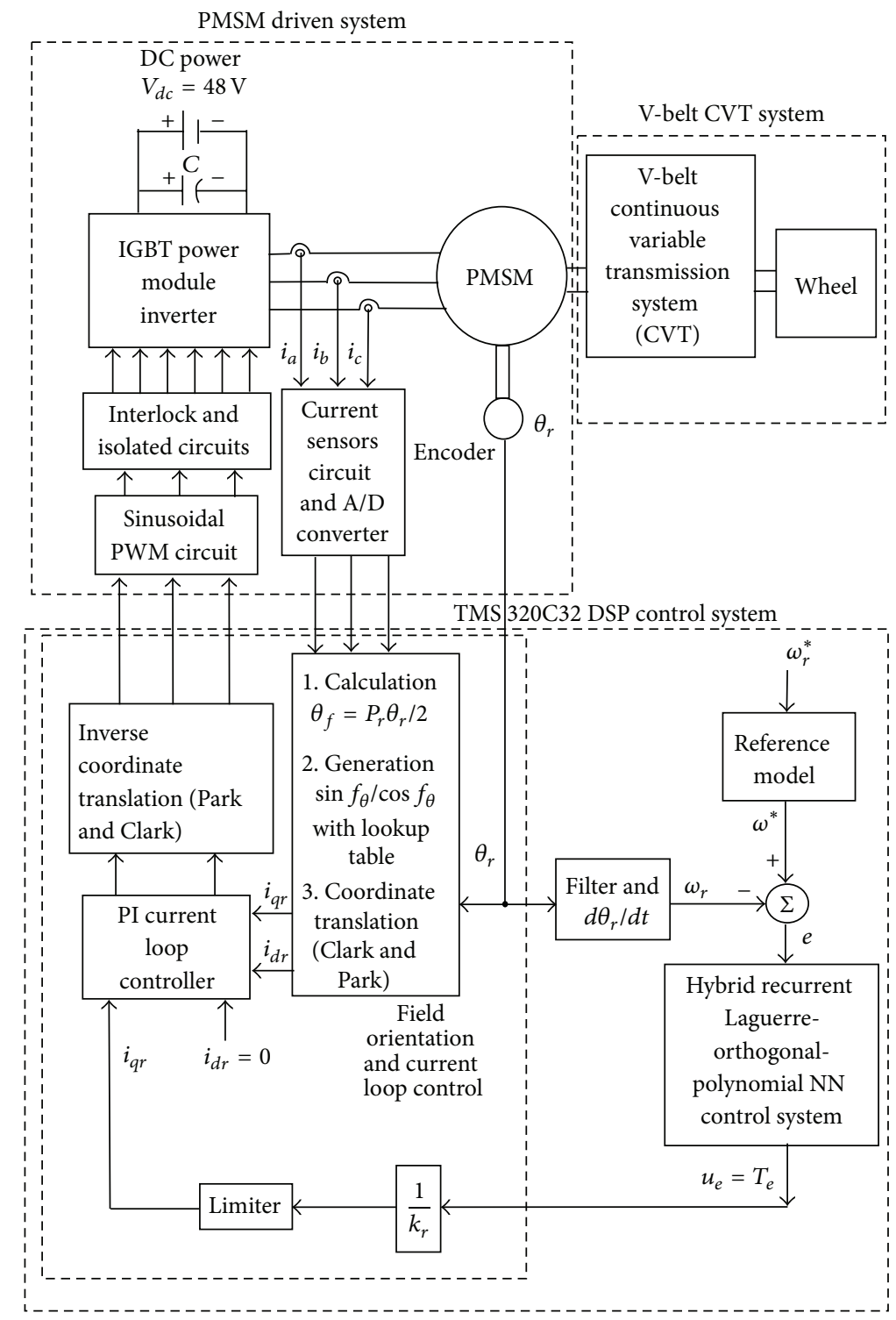

FIGURE 3: Block diagram of the PMSM driven V-belt CVT system.

system driven by PMSM. These nonlinear uncertainties cause variation of rotor inertia and friction for the V-belt CVT system driven by PMSM. For convenient hybrid recurrent Laguerre-orthogonal-polynomial NN control system design, the dynamic equation of the V-belt CVT system driven by PMSM from (2) can be rewritten as

$$
\begin{aligned}
\dot{\omega}_{r} & =-\frac{B_{r} \omega_{r}}{J_{r}}-\frac{T_{l}\left(F_{l}, v_{a}, \tau_{a}, \omega_{r}^{2}\right)}{J_{r}}+\frac{k_{r} i_{q r}}{J_{r}} \\
& =A_{a} \omega_{r}+C_{a} T_{l}\left(F_{l}, v_{a}, \tau_{a}, \omega_{r}^{2}\right)+B_{a} u_{e},
\end{aligned}
$$

in which $u_{e}=T_{e}$ is the control effort, that is, the command torque of the PMSM. $A_{a}=-B_{r} / J_{r}, B_{a}=1 / J_{r}$, and $C_{a}=-1 / J_{r}$ are three known constants. When the uncertainties including variation of system parameters and external force disturbance occur, the parameters are assumed to be bounded, that is,
$\left|A_{a} \omega_{r}\right| \leq D_{1}\left(\omega_{r}\right),\left|C_{a} T_{l}\left(F_{l}, v_{a}, \tau_{a}, \omega_{r}^{2}\right)\right| \leq D_{2}$, and $D_{3} \leq B_{a}$, where $D_{1}\left(\omega_{r}\right)$ is a known continuous function; $D_{2}$ and $D_{3}$ are two known constants. Then, the tracking error can be defined as

$$
e=\omega^{*}-\omega_{r}
$$

where $\omega^{*}$ represents the desired command rotor speed; $e$ is the tracking error between the desired rotor speed and actual rotor speed. If all parameters of the V-belt CVT system driven by PMSM including the lumped nonlinear external disturbances and parameter variation are well known, the ideal control law can be designed as

$$
u_{e}^{*}=\frac{\left[\dot{\omega}^{*}+k_{1} e-A_{a} \omega_{r}-C_{a} T_{l}\left(F_{l}, v_{a}, \tau_{a}, \omega_{r}^{2}\right)\right]}{B_{a}},
$$




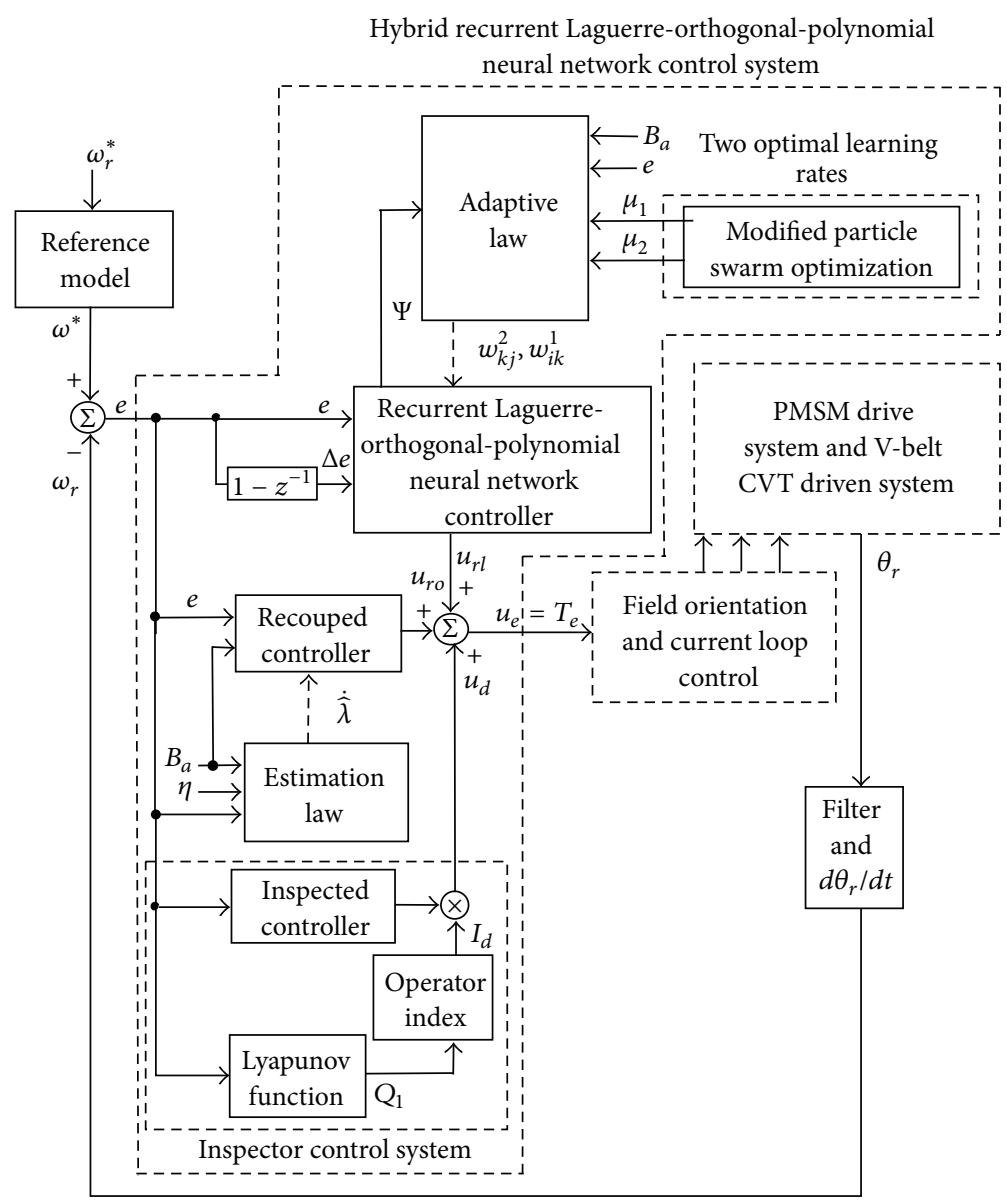

FIGURE 4: Block diagram of the hybrid recurrent Laguerre-orthogonal-polynomial NN control system.

in which $k_{1}$ is a positive constant. Substituting (6) into (4), the error dynamic equation can be obtained as follows:

$$
\dot{e}+k_{1} e=0 \text {. }
$$

The system state can track the desired trajectory gradually if $e(t) \rightarrow 0$ as $t \rightarrow \infty$ in (8). However, the hybrid recurrent Laguerre-orthogonal-polynomial NN control system is proposed to control the CVT driven by PMSM under uncertain perturbations. The configuration of the proposed hybrid recurrent Laguerre-orthogonal-polynomial NN control system is described in Figure 4.

The hybrid recurrent Laguerre-orthogonal-polynomial $\mathrm{NN}$ control system is composed of an inspector control system, a recurrent Laguerre-orthogonal-polynomial NN controller, and a recouped controller. The control law is designed as

$$
u_{e}=u_{d}+u_{\mathrm{rl}}+u_{\mathrm{ro}}
$$

where $u_{d}$ is the proposed inspected control that is able to stabilize around a predetermined bound area in the states of the controlled system; $u_{\mathrm{rl}}$ is the recurrent Laguerre-orthogonalpolynomial NN control which is as the major tracking controller. It is used to imitate an ideal control law. The recouped control $u_{\text {ro }}$ is designed to recoup the difference between the ideal control law and the recurrent Laguerre-orthogonalpolynomial NN control. Since the inspected control caused the overdone and chattering effort, the recurrent Laguerreorthogonal-polynomial NN control and the recouped control are proposed to reduce and smooth the control effort when the system states are inside the predetermined bound area. When the recurrent Laguerre-orthogonal-polynomial NN approximation properties cannot be ensured, the inspected control is able to act in this case.

For the condition of divergence of states, the design of hybrid recurrent Laguerre-orthogonal-polynomial NN control system is essential to stretch the divergent states back to the predestinated bound area. The hybrid recurrent Laguerreorthogonal-polynomial NN control system can uniformly approximate the ideal control law inside the bound area. Then stability of the hybrid recurrent Laguerre-orthogonalpolynomial NN control system can be warranted. An error dynamic equation from (5) to (9) can be acquired as

$$
\dot{e}=-k_{1} e+\left[u_{e}^{*}-u_{d}-u_{\mathrm{rl}}-u_{\mathrm{ro}}\right] B_{a} .
$$

Firstly, the inspected control $u_{d}$ can be designed as

$$
u_{d}=\frac{I_{d} \operatorname{sgn}\left(e B_{a}\right)\left[D_{1}\left(\omega_{r}\right)+D_{2}+\left|\dot{\omega}^{*}\right|+\left|k_{1} e\right|\right]}{B_{a}},
$$




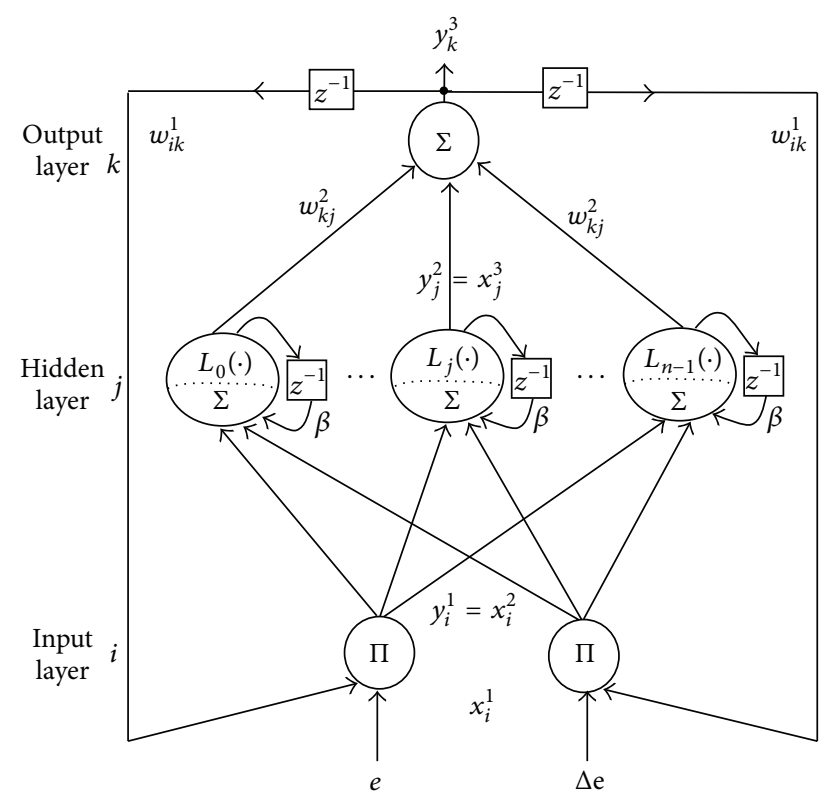

FIGURE 5: Structure of the three-layer recurrent Laguerre-orthogonal-polynomial NN.

in which $\operatorname{sgn}(\cdot)$ is a sign function. When the recurrent Laguerre-orthogonal-polynomial NN approximation properties cannot be ensured, the inspected control law is able to act in this case; that is, $I_{d}=1$. Due to the inadequate bound values, for example, $D_{1}\left(\omega_{r}\right), D_{2}, D_{3}$, and sign function, the inspected control can produce in overdone and chattering effort. Therefore, the recurrent Laguerre-orthogonalpolynomial $\mathrm{NN}$ control and the recouped control can be devised to conquer the mentioned blemish. The recurrent Laguerre-orthogonal-polynomial NN control raised to imitate the ideal control $u_{e}^{*}$. Then the recouped control posed to recoup the difference between the ideal control $u_{e}^{*}$ and the recurrent Laguerre-orthogonal-polynomial NN control $u_{\mathrm{rl}}$.

Secondly, the architecture of the proposed three-layer recurrent Laguerre-orthogonal-polynomial NN is depicted in Figure 5. It is composed of an input layer, a hidden layer, and an output layer. The activation functions and signal actions of nodes in each layer of the recurrent Laguerreorthogonal-polynomial NN can be described as follows.

First Layer: Input Layer. Each node $i$ in this layer is indicated by using $\Pi$, which multiplies by each other between each other for input signals. Then outputs signals are the results of product. The input and the output for each node $i$ in this layer are expressed as

$$
\begin{array}{r}
\operatorname{nod}_{i}^{1}=\prod_{k} x_{i}^{1}(N) w_{i k}^{1} y_{k}^{3}(N-1), \\
y_{i}^{1}=f_{i}^{1}\left(\operatorname{nod}_{i}^{1}\right)=\operatorname{nod}_{i}^{1}, \\
i=1,2 .
\end{array}
$$

The $x_{1}^{1}=\omega^{*}-\omega_{r}=e$ is the tracking error between the desired speed $\omega^{*}$ and the rotor speed $\omega_{r}$. The $x_{2}^{1}=e\left(1-z^{-1}\right)=\Delta e$ is the tracking error change. The $w_{i k}^{1}$ is the recurrent weight between output layer and input layer. The $N$ denotes the number of iterations. The $y_{k}^{3}$ is the output value of the output layer in the recurrent Laguerre-orthogonal-polynomial NN.

Second Layer: Hidden Layer. The single node $j$ th in this layer is labeled with $\Sigma$. The net input and the net output for node $j$ th of the hidden layer are expressed as

$$
\begin{array}{r}
\operatorname{nod}_{j}^{2}(N)=\sum_{i=1}^{2} x_{i}^{2}(N)+\beta y_{j}^{2}(N-1), \\
y_{j}^{2}=f_{j}^{2}\left(\operatorname{nod}_{j}^{2}\right)=L_{j}\left(\operatorname{nod}_{j}^{2}\right), \\
j=0,1, \ldots, m-1 .
\end{array}
$$

Laguerre-orthogonal polynomials [20-24] are selected for activation function of the hidden layer. The Laguerreorthogonal polynomials are denoted by $L_{n}(x)$, where $n$ is the order of expansion and $-1<x<1$ is the argument of the polynomial, $m$ is the number of nodes, and $\beta$ is the self-connecting feedback gain of the hidden layer which is selected between 0 and 1 . The zero, the first, and the second order Laguerre-orthogonal polynomials are given by $L_{0}(x)=$ $1, L_{1}(x)=1-x$, and $L_{2}(x)=x^{2}-4 x+2$, respectively. The higher order polynomials are given by $L_{3}(x)=-x^{3}+$ $9 x^{2}-18 x+6$ and $L_{4}(x)=\left(35 x^{4}-30 x^{2}+3\right) / 8$. The higher order Laguerre-orthogonal polynomials may be generated by the recursive formula given by $L_{n+1}(x)=\left[(2 n+1-x) L_{n}(x)-\right.$ $\left.n L_{n-1}(x)\right] /(n+1)$.

Third Layer: Output Layer. The single node $k$ th in this layer is labeled with $\Sigma$. It computes the overall output as the 
summation of all input signals. The net input and the net output for node $k$ th in this layer are expressed as

$$
\operatorname{nod}_{k}^{3}=\sum_{j=0}^{m-1} w_{k j}^{2} x_{j}^{3}(N), \quad y_{k}^{3}=f_{k}^{3}\left(\operatorname{nod}_{k}^{3}\right)=\operatorname{nod}_{k}^{3}, \quad k=1,
$$

where $w_{k j}^{2}$ is the connective weight between the hidden layer and the output layer; $f_{k}^{3}$ is the activation function, which is selected as a linear function; $x_{j}^{3}(N)=y_{j}^{2}(N)$ represents the $j$ th input to the node of output layer. The output value of the recurrent Laguerre-orthogonal-polynomial NN can be represented as $y_{k}^{3}(N)=u_{\mathrm{rl}}$. Then the output value of the recurrent Laguerre-orthogonal-polynomial $\mathrm{NN}, u_{\mathrm{rl}}$, can be denoted as

$$
u_{\mathrm{rl}}=\boldsymbol{\Theta}^{T} \Psi
$$

in which $\Theta=\left[\begin{array}{lll}w_{10}^{2} & \cdots & w_{1, m-1}^{2}\end{array}\right]^{T}$ is the adjustable weight parameters vector between the hidden layer and the output layer of the recurrent Laguerre-orthogonal-polynomial NN. $\Psi=\left[\begin{array}{lll}x_{0}^{3} & \cdots & x_{m-1}^{3}\end{array}\right]^{T}$ is the inputs vector in the output layer of the recurrent Laguerre-orthogonal-polynomial $\mathrm{NN}$, in which $x_{j}^{3}$ is determined by the selected Laguerre-orthogonalpolynomials.

Thirdly, in order to evolve the recouped control $u_{\text {ro }}$, a minimum approximation error $\delta$ is defined as

$$
\delta=u_{e}^{*}-u_{\mathrm{rl}}^{*}=u_{e}^{*}-\left(\boldsymbol{\Theta}^{*}\right)^{T} \boldsymbol{\Psi}
$$

in which $\Theta^{*}$ is an ideal weight vector to reach minimum approximation error. It is assumed that absolute value of $\delta$ is less than a small positive value $\lambda$; that is, $|\delta|<\lambda$. Then, the error dynamic equation from (10) can be rewritten as

$$
\begin{aligned}
\dot{e} & =-k_{1} e+\left[\left(u_{e}^{*}-u_{\mathrm{rl}}\right)-u_{\mathrm{rl}}-u_{d}\right] B_{a} \\
& =-k_{1} e+\left[\left(u_{e}^{*}-u_{\mathrm{rl}}^{*}+u_{\mathrm{rl}}^{*}-u_{\mathrm{rl}}\right)-u_{\mathrm{ro}}-u_{d}\right] B_{a} \\
& =-k_{1} e+\left[\left(u_{e}^{*}-u_{\mathrm{rl}}^{*}\right)+\left(\boldsymbol{\Theta}^{*}\right)^{T} \Psi-(\boldsymbol{\Theta})^{T} \Psi-u_{\mathrm{ro}}-u_{d}\right] B_{a} \\
& =-k_{1} e+\left[\delta+\left(\Theta^{*}-\Theta\right)^{T} \Psi-u_{\mathrm{ro}}-u_{d}\right] B_{a} .
\end{aligned}
$$

Then, the Lyapunov function is selected as

$$
L_{1}(t)=\frac{e^{2}}{2}+\frac{\left(\boldsymbol{\Theta}^{*}-\boldsymbol{\Theta}\right)^{T}\left(\boldsymbol{\Theta}^{*}-\boldsymbol{\Theta}\right)}{\left(2 \mu_{1}\right)}+\frac{\tilde{\lambda}^{2}}{2 \eta}
$$

in which $\mu_{1}$ is a learning rate, $\eta$ is an adaptation gain, and $\tilde{\lambda}=\widehat{\lambda}-\lambda$ is the bound estimated error. Differentiating the
Lyapunov function with respect to $t$ and using (16), then (17) can be rewritten as

$$
\begin{aligned}
\dot{L}_{1}(t)= & \dot{e} e-\frac{\left(\Theta^{*}-\boldsymbol{\Theta}\right)^{T} \dot{\Theta}}{\mu_{1}}+\frac{\tilde{\lambda} \dot{\hat{\lambda}}}{\eta} \\
= & -k_{1} e+\left[\delta-u_{\mathrm{ro}}-u_{d}\right] B_{a} e \\
& +\left(\boldsymbol{\Theta}^{*}-\boldsymbol{\Theta}\right)^{T} \boldsymbol{\Psi} B_{a} e-\frac{\left(\boldsymbol{\Theta}^{*}-\boldsymbol{\Theta}\right)^{T} \dot{\Theta}}{\mu_{1}}+\frac{\tilde{\lambda} \dot{\hat{\lambda}}}{\eta} .
\end{aligned}
$$

In order to obtain $\dot{L}_{1} \leq 0$, the adaptive law $\dot{\Theta}$ and the recouped controller $u_{\text {ro }}$ with estimation law $\widehat{\lambda}$ can be designed as follows:

$$
\begin{gathered}
\dot{\Theta}=\mu_{1} \Psi B_{a} e, \\
u_{\mathrm{ro}}=\widehat{\lambda} \operatorname{sgn}\left(B_{a} e\right), \\
\dot{\hat{\lambda}}=\eta\left|B_{a} e\right| .
\end{gathered}
$$

Substituting (11), (20), and (21) into (19) and using (11) with $I_{d}=0$, then (19) can be represented as

$$
Q_{1}(t)=-k_{1} e^{2}+\left(\delta-\hat{\lambda} \operatorname{sgn}\left(B_{a} e\right)\right) B_{a} e+\frac{\tilde{\lambda} \dot{\hat{\lambda}}}{\eta}
$$

Substituting (22) into (23), then (23) can be obtained as

$$
\dot{Q}_{1}(t) \leq-k_{1} e^{2}+\{|\delta|-\lambda\}\left|B_{a} e\right| \leq-k_{1} e^{2} \leq 0 .
$$

From (24), the $\dot{Q}_{1}(t)$ is a negative semidefinite; that is, $Q_{1}(t) \leq$ $Q_{1}(0)$. It implies that $e$ and $\left(\Theta^{*}-\Theta\right)$ are bounded. In addition, the function is defined as

$$
\varepsilon(t)=-\dot{Q}_{1}(t)=k_{1} e^{2} .
$$

Integrating (25) with respect to $t$, then

$$
\int_{0}^{t} \varepsilon(\tau) d \tau=\int_{0}^{t}\left[-\dot{Q}_{1}(t)\right] d t=Q_{1}(0)-Q_{1}(t)
$$

Due to the fact that $Q_{1}(0)$ is bounded and $Q_{1}(t)$ is nonincreasing and bounded, then

$$
\lim _{t \rightarrow \infty} \int_{0}^{t} \varepsilon(\tau) d \tau<\infty
$$

Differentiating (25) with respect to $t$ gives

$$
\dot{\varepsilon}(t)=2 k_{1} e \dot{e} .
$$

Due to the fact that all the variables in the right side of (17) are bounded. It implies that $\dot{e}$ is also bounded. Then, $\varepsilon(t)$ is a uniformly continuous function [51, 52]. It is denoted that $\lim _{t \rightarrow \infty} \varepsilon(t)=0$ by using Barbalat's lemma [51, 52]. Therefore, $e(t) \rightarrow 0$ as $t \rightarrow \infty$. Furthermore, In order ${ }^{\sim}$ to avoid chattering phenomenon of recouped controller $u_{\text {ro }}$, 
the sign function $\operatorname{sgn}\left(B_{a} e\right)$ can be replaced by the equation $B_{a} e /\left(\left|B_{a} e\right|+\rho\right)$, where

$$
\rho= \begin{cases}\rho_{0}, & \left|B_{a} e\right|<\tau \\ 0, & \left|B_{a} e\right| \geq \tau,\end{cases}
$$

in which $\rho_{0}$ and $\tau$ are positive constants.

According to Lyapunov stability theorem and the gradient descent method, an online parameter training methodology of the recurrent Laguerre-orthogonal-polynomial NN can be derived and trained effectively. Then the parameter of adaptive law $\dot{\Theta}$ can be computed by the gradient descent method. The adaptive law $\dot{\Theta}$ shown in (20) calls for a proper choice of the learning rate. For a small value of learning rate, the convergence of controller parameter can be guaranteed but the convergent speed is very slow. In order to achieve the fast convergence of output tracking error, the modified PSO is used to obtain optimal learning rate for training parameters of the recurrent Laguerre-orthogonal-polynomial NN. First, the parameter of adaptive law $\dot{\Theta}$ shown in (20) can be rewritten as

$$
\dot{w}_{k j}^{2}=\mu_{1} x_{j}^{3} B_{a} e .
$$

The central part of the training algorithm for the recurrent Laguerre-orthogonal-polynomial NN is concerned with how to obtain recursively a gradient vector in which each element in the training algorithm is defined as the derivative of an energy function with respect to a parameter of the network. This is done by means of the chain rule, because the gradient vector is calculated in the direction opposite to the flow of the output of each node. In order to describe the online training algorithm of the recurrent Laguerre-orthogonal-polynomial $\mathrm{NN}$, a cost function is defined as

$$
V_{1}=\frac{e^{2}}{2} .
$$

According to the gradient descent method, the adaptive law of the weight also can be represented as

$$
\dot{w}_{k j}^{2}=-\mu_{1} \frac{\partial V_{1}}{\partial w_{k j}^{2}}=-\mu_{1} \frac{\partial V_{1}}{\partial y_{k}^{3}} \frac{\partial y_{k}^{3}}{\partial \operatorname{nod}_{k}^{3}} \frac{\partial \operatorname{nod}_{k}^{3}}{\partial w_{k j}^{2}}=-\mu_{1} \frac{\partial V_{1}}{\partial y_{k}^{3}} x_{j}^{3} .
$$

Comparing (30) with (32) yields $\partial V_{1} / \partial y_{k}^{3}=-e B_{a}$. Then, the convergence analysis in the following theorem is to derive specific learning rate to assure convergence of the output tracking error.

The adaptation law of recurrent weight $w_{i k}^{1}$ using the gradient descent method can be updated as

$$
\begin{aligned}
\dot{w}_{i k}^{1} & =-\mu_{2} \frac{\partial V_{1}}{\partial u_{\mathrm{re}}} \frac{\partial u_{\mathrm{re}}}{\partial y_{k}^{3}} \frac{\partial y_{k}^{3}}{\partial y_{j}^{2}} \frac{\partial y_{j}^{2}}{\partial \operatorname{nod}_{j}^{2}} \frac{\partial \operatorname{nod}_{j}^{2}}{\partial y_{i}^{1}} \frac{\partial y_{i}^{1}}{\partial \operatorname{nod}_{i}^{1}} \frac{\partial \operatorname{nod}_{i}^{1}}{\partial w_{i k}^{1}} \\
& =\mu_{2} B_{a} e w_{k j}^{2} L_{j}(\cdot) x_{i}^{1}(N) y_{k}^{3}(N-1)
\end{aligned}
$$

in which $\mu_{2}$ is a learning rate. Then, the convergence analysis in the following theorem is to derive specific learning rate to assure convergence of the output tracking error. In order to obtain better learning rate, therefore, the modified PSO is proposed to search for a better learning rate of the weights of the recurrent Laguerre-orthogonal-polynomial NN.

The PSO [30-42], which has three parameters as two acceleration coefficients $c_{1}, c_{2}$ and inertia weight $\gamma(n)$, has a significant impact on performance of the algorithm, especially the impact of inertia weight. The impact is different on different conditions and is also different at different times under the same condition. The PSO with larger inertia weight has a faster convergence speed and works well in global search, while the PSO with smaller inertia weight can reach a more accurate optimum value but only works well in local search. Now dynamic inertia weight modification is used to train the appropriate value of $\gamma(n)$, in order to coordinate between search accuracy and search speed. The modified PSO algorithm depends in its implementation in the following two relations:

$$
\begin{gathered}
v_{j}(n+1)=\gamma(n) v_{j}(n) \\
+\alpha(n)\left[c_{1} \varphi_{1}\left(P_{b j}-\mu_{m j}(n)\right)\right. \\
\left.+c_{2} \varphi_{2}\left(P_{g j}-\mu_{m j}(n)\right)\right], \quad m=1,2, \\
\mu_{m j}(n+1)=\mu_{m j}(n)+v_{j}(n+1), \quad m=1,2, \\
\gamma(n)=\gamma_{0}+\varphi_{3}\left(1-\gamma_{0}\right), \\
\alpha(n)=\alpha_{0}+\frac{\alpha_{1} n}{T},
\end{gathered}
$$

where $n=1,2, \ldots, N_{\max }$ indicates the iterations; $\mu_{m j}(n)$ is the current position of particle $P_{j}$ in hyperspace and with respect to learning rate $\mu_{m}(n), m=1,2 ; v_{j}(n)$ is the current speed of particle $j ; \gamma(n)$ is the inertia weight with respective to $n ; c_{1}$ and $c_{2}$ are acceleration positive constants; $\varphi_{1}, \varphi_{2}$, and $\varphi_{3}$ are random numbers obtained from a uniform random distribution function in the interval $[0,1] ; \gamma_{0}$ is the initial positive constant in the interval $[0,1] ; \alpha_{0}$ and $\alpha_{1}$ are the initial positive constants in the interval $[0,1] ; T$ denotes the number of generations; $P_{b j}$ and $P_{g j}$ represent the best previous position of the $j$ th particle and the position of the best particle among all particles in the population, respectively. Usually, $\gamma(n)$ is modified gradually in descending within the limit of $0.8<\gamma(n)<1.4$ [40], so that search space can be changed steadily from the global to the local. Decreasing inertia weight particle swarm optimization is a topical algorithm, of which inertia weight $\gamma(n)$ decreases linearly from 0.9 to $0.4[38,39]$. Some scholars propose the increasing inertia weight particle swarm optimization of which inertia weight $\gamma(t)$ increases linearly from 0.4 to $0.9[40,41] . \alpha(n)$ is the constriction factor introduced by Eberhart and Shi $[38,39]$ to avoid the swarm from premature convergence and to ensure stability of the system.

\section{Experimental Results}

The whole system of the DSP-based control system for the PMSM driven V-belt CVT system is shown in Figure 1. The 


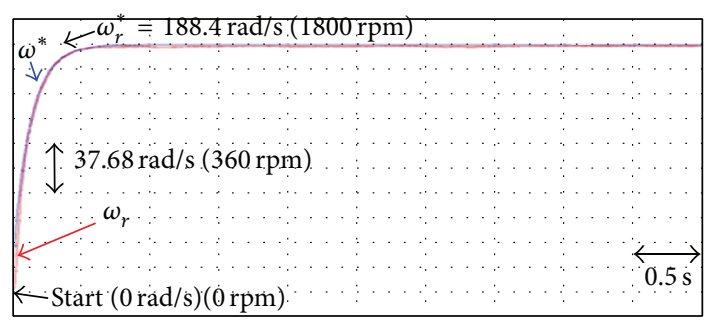

(a)

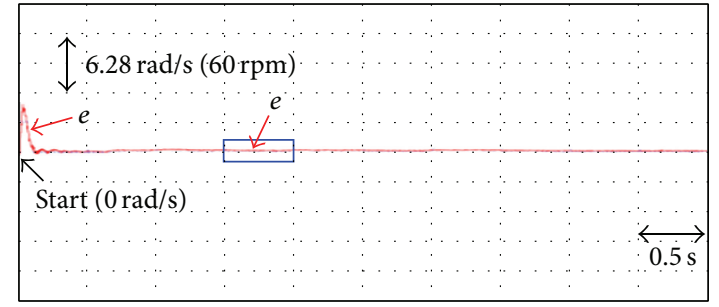

(b)

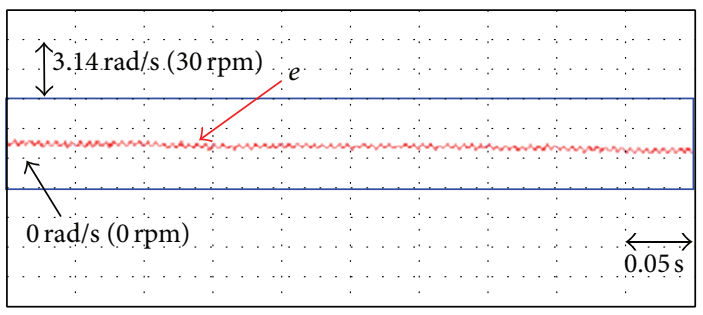

(c)

FIGURE 6: Experimental results of the PMSM driven V-belt CVT system using the well-known PI controller at $188.4 \mathrm{rad} / \mathrm{s}$ (1800 rpm) case under the lumped nonlinear external disturbances with parameter variation $T_{l}=\Delta T_{p}+T_{\text {un }}$ : (a) tracking response of command rotor speed $\omega_{r}^{*}$, desired command rotor speed $\omega^{*}$, and measured rotor speed $\omega_{r}$; (b) response of tracking error $e$; (c) response of tracking error $e$ amplification.

control algorithm was executed by a TMS320C32 DSP control system including four channels of D/A, eight channels of programmable PWM, and one encoder interface circuit. The IGBT power module voltage source inverter is executed by current-controlled SPWM with a switching frequency of $15 \mathrm{kHz}$. The specification of PMSM is a three-phase $48 \mathrm{~V}$, $750 \mathrm{~W}, 16.5 \mathrm{~A}$, and $3600 \mathrm{rpm}$. The parameters of the PMSM are given as follows as $R_{r}=2.5 \Omega, L_{d r}=L_{q r}=6.53 \mathrm{mH}$, $\bar{J}_{r}=62.15 \times 10^{-3} \mathrm{Nms}, \bar{B}_{r}=6.18 \times 10^{-3} \mathrm{Nms} / \mathrm{rad}$, and $k_{r}=0.86 \mathrm{Nm} / \mathrm{A}$ by using open circuit test, short test, rotor block test, and loading test. Owing to inherent uncertainty in V-belt CVT system (e.g., the lumped nonlinear external disturbances and parameter variations) and output current limitation of battery capacity, the PMSM can only operate at $376.8 \mathrm{rad} / \mathrm{s}(3600 \mathrm{rpm})$ to avoid burning IGBT modules for V-belt CVT system at high speed perturbation.

To show the control performance of the proposed hybrid recurrent Laguerre-orthogonal-polynomial NN control system, two cases are provided in the experimentation. One is the $188.4 \mathrm{rad} / \mathrm{s}(1800 \mathrm{rpm})$ case under the lumped nonlinear external disturbances with parameter variation $T_{l}=\Delta T_{p}+T_{\text {un }}$ and the other is the $376.8 \mathrm{rad} / \mathrm{s}(3600 \mathrm{rpm})$ case under the lumped nonlinear external disturbances with twice parameter variations $T_{l}=2 \Delta T_{p}+T_{\text {un }}$. In order to achieve good transient and steady-state control performance all gains of the well-known PI controller are $k_{\mathrm{ps}}=13.5$ and $k_{\mathrm{is}}=k_{\mathrm{ps}} / T_{\mathrm{is}}=$ 1.8 through some heuristic knowledge [47-50] on the tuning of the PI controller at $188.4 \mathrm{rad} / \mathrm{s}(1800 \mathrm{rpm})$ case under the lumped nonlinear external disturbances with parameter variation $T_{l}=\Delta T_{p}+T_{\text {un }}$ for the speed tracking. The experimental results of the well-known PI controller for the V-belt CVT system driven by PMSM at $188.4 \mathrm{rad} / \mathrm{s}$ (1800 rpm) case under the lumped nonlinear external disturbances with parameter variation $T_{l}=\Delta T_{p}+T_{\text {un }}$ and $376.8 \mathrm{rad} / \mathrm{s}(3600 \mathrm{rpm})$ case under the lumped nonlinear external disturbances with twice parameter variations $T_{l}=2 \Delta T_{p}+T_{\text {un }}$ are shown in Figures 6,7 , and 8 , where tracking responses of the command rotor speed $\omega_{r}^{*}$, the desired command rotor speed $\omega^{*}$, and the measured rotor speed $\omega_{r}$ are shown in Figures 6(a) and $7(\mathrm{a})$; tracking responses of the speed error $e$ are shown in Figures 6(b) and 7(b); tracking responses of the speed error $e$ amplification are shown in Figures 6(c) and 7(c). Since the low speed operation is the same as the nominal case due to smaller disturbance, the response of speed shown in Figure 6(a) has better tracking performance. Moreover, the degenerate tracking responses of speed shown in Figure 7(a) is obvious due to bigger nonlinear disturbance (e.g., rolling resistance, wind resistance, and parameter variation) at high speed operation. From the experimental results, sluggish tracking responses of speed and current are obtained for the V-belt CVT system driven by PMSM using the wellknown PI controller. The linear controller has the weak robustness under bigger nonlinear disturbance because of no appropriate gains tuning or no degenerate nonlinear effect. In addition, responses of the command electromagnetic torque $T_{e}$ at $188.4 \mathrm{rad} / \mathrm{s}(1800 \mathrm{rpm})$ case under the lumped nonlinear external disturbances with parameter variation $T_{l}=\Delta T_{p}+T_{\text {un }}$ and $376.8 \mathrm{rad} / \mathrm{s}$ (3600 rpm) case under the lumped nonlinear external disturbances with twice parameter variations $T_{l}=$ $2 \Delta T_{p}+T_{\text {un }}$ are shown in Figures 8(a) and 8(b), respectively. The dynamic response of command electromagnetic torque $T_{e}$ results in great torque ripple due to $\mathrm{V}$-belt CVT system with nonlinear disturbance, such as $\mathrm{V}$-belt shaking friction and action frictions between primary pulley and second pulley.

The control gains of the proposed hybrid recurrent Laguerre-orthogonal-polynomial NN control system are $\eta=$ $0.1, \lambda=0.3$. All control gains of the hybrid recurrent Laguerre-orthogonal-polynomial NN control system are chosen to achieve the best transient control performance 


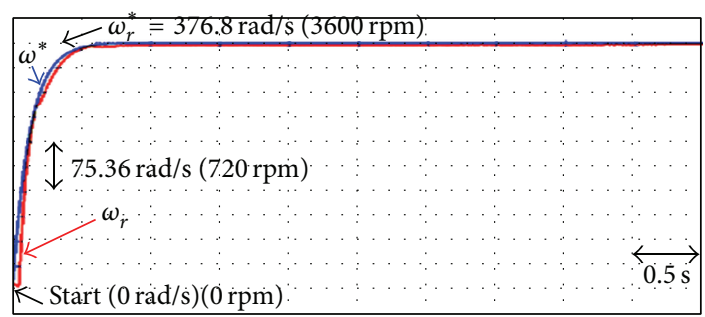

(a)

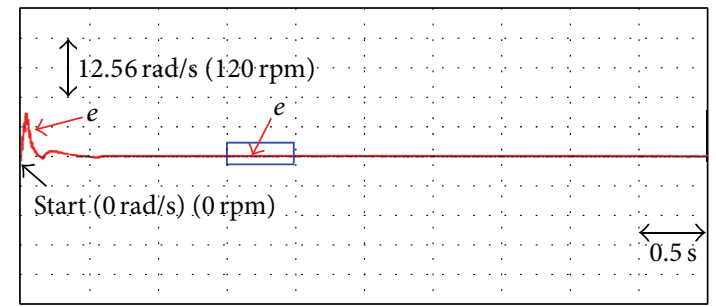

(b)

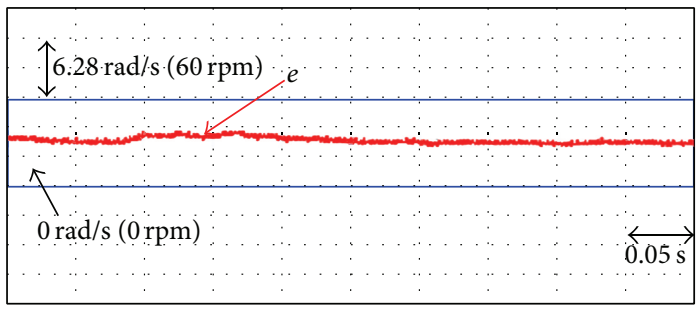

(c)

FIGURE 7: Experimental results of the PMSM driven V-belt CVT system using the well-known PI controller at $376.8 \mathrm{rad} / \mathrm{s}$ ( $3600 \mathrm{rpm}$ ) case under the lumped nonlinear external disturbances with twice parameter variations $T_{l}=2 \Delta T_{p}+T_{\text {un }}$ : (a) tracking response of command rotor speed $\omega_{r}^{*}$, desired command rotor speed $\omega^{*}$, and measured rotor speed $\omega_{r}$; (b) response of tracking error $e$; (c) response of tracking error $e$ amplification.

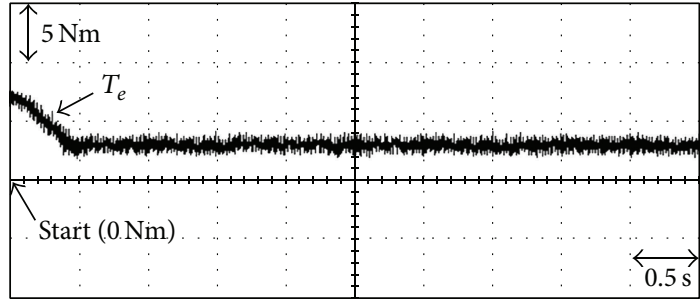

(a)

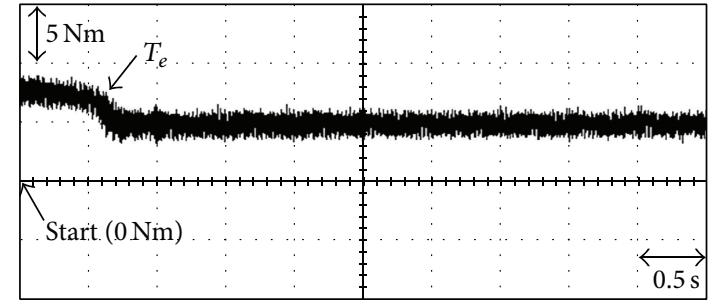

(b)

FIGURE 8: Experimental results of the PMSM driven V-belt CVT system using the well-known PI controller: (a) response of electromagnetic torque $T_{e}$ at $188.4 \mathrm{rad} / \mathrm{s}(1800 \mathrm{rpm})$ case under the lumped nonlinear external disturbances with parameter variation $T_{l}=\Delta T_{p}+T_{\text {un }} ;(\mathrm{b})$ response of electromagnetic torque $T_{e}$ at $376.8 \mathrm{rad} / \mathrm{s}(3600 \mathrm{rpm})$ case under the lumped nonlinear external disturbances with twice parameter variations $T_{l}=2 \Delta T_{p}+T_{\text {un }}$.

in experimentation considering the requirement of stability. The parameter adjustment process remains continually active for the duration of the experimentation. The structure of the recurrent Laguerre-orthogonal-polynomial NN controller has 2 nodes, 3 nodes, and 1 node in the input layer, the hidden layer, and the output layer, respectively. The experimental results of the proposed hybrid recurrent Laguerre-orthogonal-polynomial NN control system for the $\mathrm{V}$-belt CVT system driven by PMSM at $188.4 \mathrm{rad} / \mathrm{s}$ (1800 rpm) case under the lumped nonlinear external disturbances with parameter variation $T_{l}=\Delta T_{p}+T_{\text {un }}$ and $376.8 \mathrm{rad} / \mathrm{s}$ (3600 rpm) case under the lumped nonlinear external disturbances with twice parameter variations $T_{l}=2 \Delta T_{p}+T_{\text {un }}$ are shown in Figures 9, 10, and 11, where tracking responses of the command rotor speed $\omega_{r}^{*}$, the desired command rotor speed $\omega^{*}$, and the measured rotor speed $\omega_{r}$ are shown in Figures 9(a) and 10(a); tracking responses of the speed error $e$ are shown in Figures 9(b) and 10(b); tracking responses of the speed error $e$ amplification are shown in Figures 9(c) and 10(c). Since the low speed operation is the same as the nominal case due to smaller disturbance, the speed tracking response shown in Figure 9(a) has better tracking performance. The better speed tracking response is shown in Figure 10(a) under the occurrence of bigger lumped nonlinear external disturbances and parameter variation. From the experimental results, the accurate tracking performance is obtained for the V-belt CVT system driven by PMSM using the hybrid recurrent Laguerre-orthogonal-polynomial $\mathrm{NN}$ control system owing to the online adaptive mechanism of the recurrent Laguerre-orthogonal-polynomial NN and action of the recouped controller. In addition, responses of the command electromagnetic torque $T_{e}$ at $188.4 \mathrm{rad} / \mathrm{s}$ $(1800 \mathrm{rpm})$ case under the lumped nonlinear external disturbances with parameter variation $T_{l}=\Delta T_{p}+T_{\text {un }}$ and $376.8 \mathrm{rad} / \mathrm{s}$ (3600 rpm) case under the lumped nonlinear external disturbances with twice parameter variations $T_{l}=$ $2 \Delta T_{p}+T_{\text {un }}$ are shown in Figures 11(a) and 11(b), respectively. In addition, in order to decrease inertia weight particle swarm 


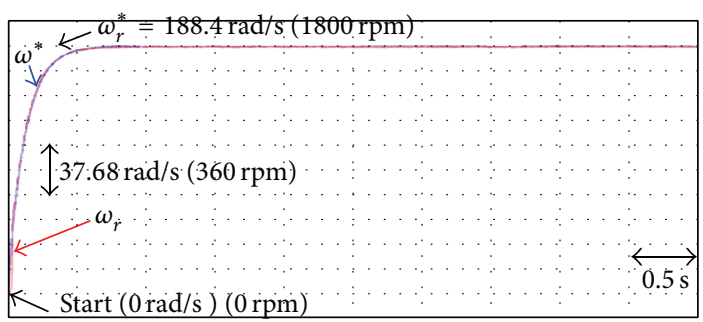

(a)

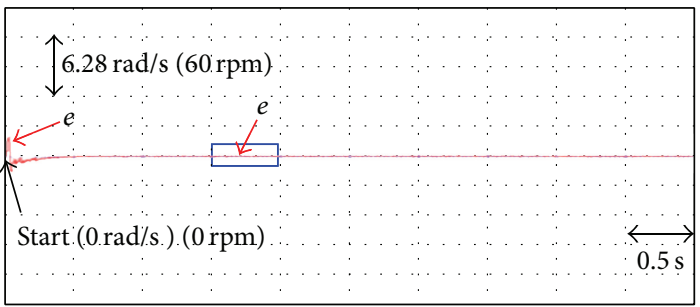

(b)

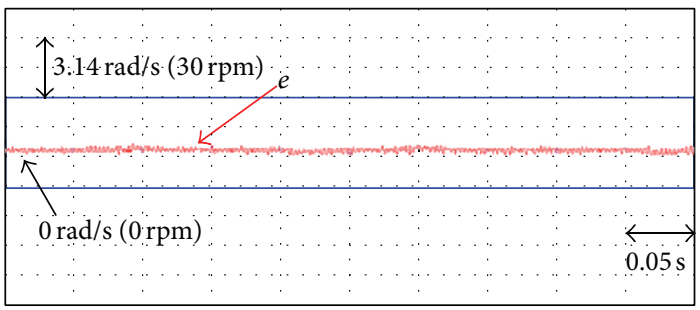

(c)

FiguRE 9: Experimental results of the PMSM driven V-belt CVT system using the hybrid recurrent Laguerre-orthogonal-polynomial NN control system at $188.4 \mathrm{rad} / \mathrm{s}(1800 \mathrm{rpm})$ case under the lumped nonlinear external disturbances with parameter variation $T_{l}=\Delta T_{p}+T_{\text {un }}$ : (a) tracking response of command rotor speed $\omega_{r}^{*}$, desired command rotor speed $\omega^{*}$, and measured rotor speed $\omega_{r}$; (b) response of tracking error $e$; (c) response of tracking error $e$ amplification.

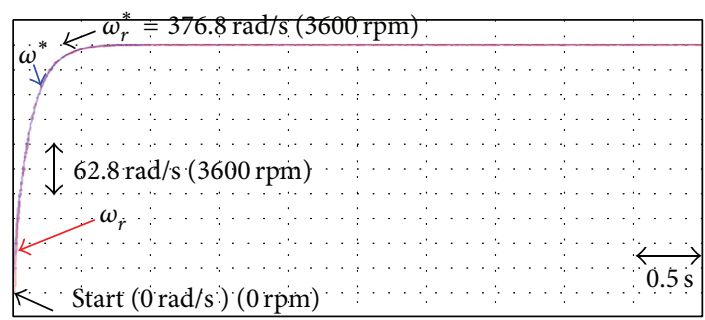

(a)

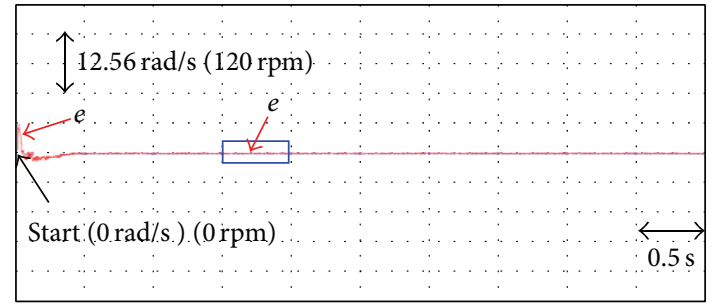

(b)

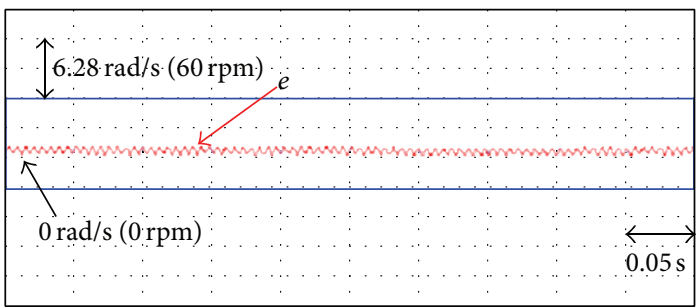

(c)

FIgURE 10: Experimental results of the PMSM driven V-belt CVT system using the hybrid recurrent Laguerre-orthogonal-polynomial NN control system at $376.8 \mathrm{rad} / \mathrm{s}(3600 \mathrm{rpm})$ under the lumped nonlinear external disturbances with twice parameter variations $T_{l}=2 \Delta T_{p}+T_{\text {un }}$ : (a) tracking response of command rotor speed $\omega_{r}^{*}$, desired command rotor speed $\omega^{*}$, and measured rotor speed $\omega_{r}$; (b) response of tracking error $e$; (c) response of tracking error $e$ amplification.

optimization, the initial coefficient $\gamma_{0}$ of inertia weight is set as 0.4. Two initial coefficients $\alpha_{0}$ and $\alpha_{1}$ of the constriction factor are set as 0.3 to avoid the swarm from premature convergence and to ensure stability of the system. Thus, the convergence responses of two learning rates $\mu_{1}$ and $\mu_{2}$ of the recurrent Laguerre-orthogonal-polynomial $\mathrm{NN}$ using modified PSO at $188.4 \mathrm{rad} / \mathrm{s}(1800 \mathrm{rpm})$ case under the lumped nonlinear external disturbances with parameter variation $T_{l}=\Delta T_{p}+T_{\text {un }}$ are shown in Figures 12(a) and 12(b), respectively. The convergence responses of two learning rates $\mu_{1}$ and $\mu_{2}$ of the recurrent Laguerre-orthogonal-polynomial NN using modified PSO at $376.8 \mathrm{rad} / \mathrm{s}$ (3600 rpm) case under the lumped nonlinear external disturbances with twice parameter variations $T_{l}=2 \Delta T_{p}+T_{\text {un }}$ are shown in Figures 13(a) and 13(b), respectively. The convergence response of two learning rates $\mu_{1}$ and $\mu_{2}$ of the recurrent Laguerre-orthogonal-polynomial NN using modified PSO have faster convergence speed than the fixed learning 


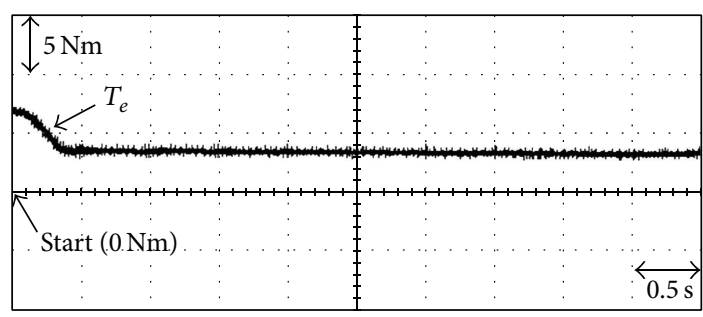

(a)

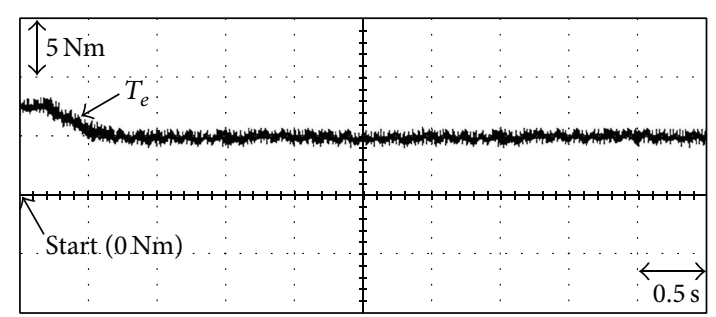

(b)

FIgURE 11: Experimental results of the PMSM driven V-belt CVT system using the hybrid recurrent Laguerre-orthogonal-polynomial NN control system: (a) response of electromagnetic torque $T_{e}$ at $188.4 \mathrm{rad} / \mathrm{s}(1800 \mathrm{rpm})$ case under the lumped nonlinear external disturbances with parameter variation $T_{l}=\Delta T_{p}+T_{\text {un }}$; (b) response of electromagnetic torque $T_{e}$ at $376.8 \mathrm{rad} / \mathrm{s}(3600 \mathrm{rpm})$ case under the lumped nonlinear external disturbances with twice parameter variations $T_{l}=2 \Delta T_{p}+T_{\text {un }}$.

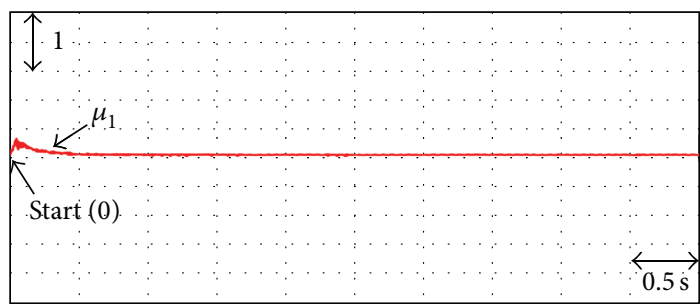

(a)

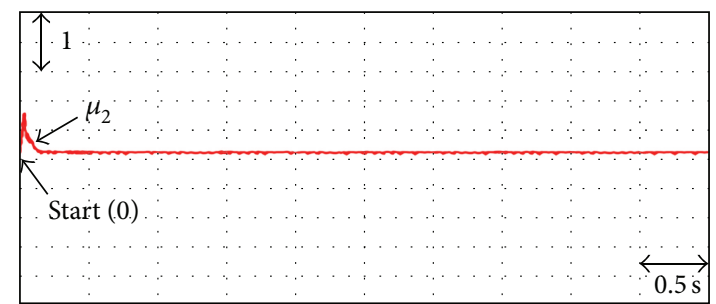

(b)

FIGURE 12: Experimental results of the recurrent Laguerre-orthogonal-polynomial NN using modified PSO at $188.4 \mathrm{rad} / \mathrm{s}$ (1800 rpm) case under the lumped nonlinear external disturbances with parameter variation $T_{l}=\Delta T_{p}+T_{\mathrm{un}}$ : (a) the convergence response of learning rates $\mu_{1}$; (b) the convergence response of learning rates $\mu_{2}$.

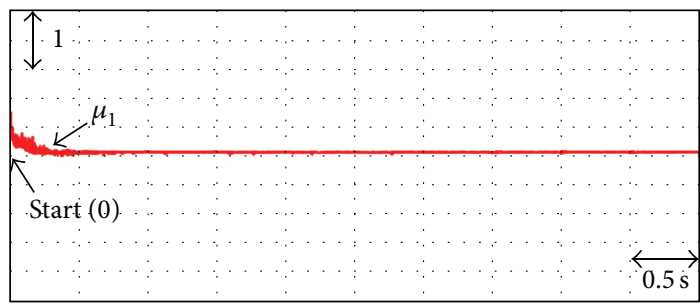

(a)

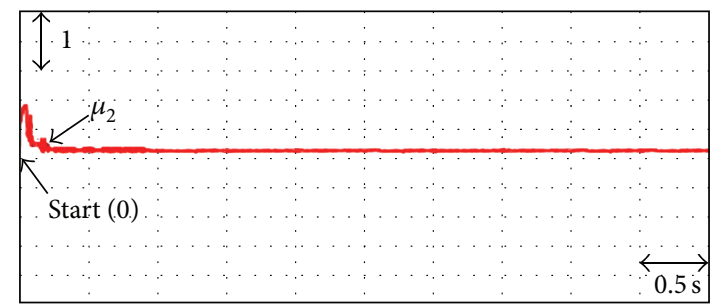

(b)

FIGURE 13: Experimental results of the recurrent Laguerre-orthogonal-polynomial NN using modified PSO at $376.8 \mathrm{rad} / \mathrm{s}$ (3600 rpm) case under the lumped nonlinear external disturbances with twice parameter variations $T_{l}=2 \Delta T_{p}+T_{\text {un }}$ : (a) the convergence response of learning rates $\mu_{1}$; (b) the convergence response of learning rates $\mu_{2}$.

rate of the recurrent Laguerre-orthogonal-polynomial NN. The dynamic response of command electromagnetic torque $T_{e}$ results lower torque ripple by on-line adjusted of the recurrent Laguerre-orthogonal-polynomial NN to cope with the high-frequency unmodeled dynamic of the V-belt CVT system with nonlinear disturbance, such as V-belt shaking friction and action frictions between primary pulley and second pulley. Therefore, these results show that the hybrid recurrent Laguerre-orthogonal-polynomial NN control system has better control performance than the well-known PI controller under high speed perturbation for the V-belt CVT system driven by PMSM. Moreover, the numerical results of the iteration performance for two learning rates $\mu_{1}$ and $\mu_{2}$ by means of modified PSO method from $0 \mathrm{~s}$ to $0.6 \mathrm{~s}$ with
240 iterations (runs) at $188.4 \mathrm{rad} / \mathrm{s}(1800 \mathrm{rpm})$ case under the lumped nonlinear external disturbances with parameter variation $T_{l}=\Delta T_{p}+T_{\text {un }}$ and $376.8 \mathrm{rad} / \mathrm{s}$ (3600 rpm) case under the lumped nonlinear external disturbances with twice parameter variations $T_{l}=2 \Delta T_{p}+T_{\text {un }}$ are shown in Figures 14 and 15, respectively. In all cases, the numerical results of the iteration performance show that modified PSO can reach the global optimum effectively and avoid premature convergence in less than 100 iterations. From above, it can be easily observed that the proposed modified PSO can provide a more accurate optimal solution and converges to the criteria with a greater probability.

The measured rotor speed responses due to step disturbance torque are given finally. The condition under $T_{l}=$ 


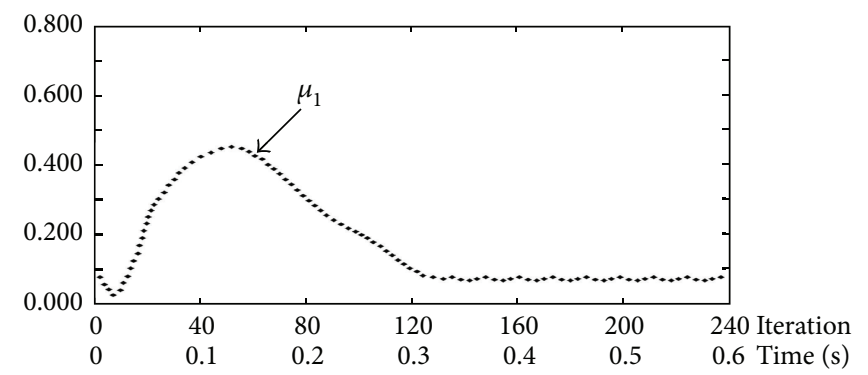

(a)

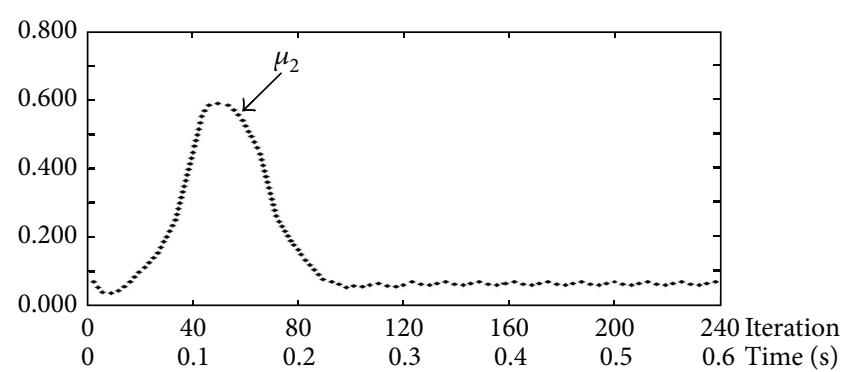

(b)

FIGURE 14: Numerical results of the iteration performance for two learning rates in the recurrent Laguerre-orthogonal-polynomial NN by means of modified PSO from $0 \mathrm{~s}$ to $0.6 \mathrm{~s}$ with 240 iterations (runs) at $188.4 \mathrm{rad} / \mathrm{s}(1800 \mathrm{rpm})$ case under the lumped nonlinear external disturbances with parameter variation $T_{l}=\Delta T_{p}+T_{\text {un }}$ : (a) response of learning rates $\mu_{1}$; (b) response of learning rates $\mu_{2}$.

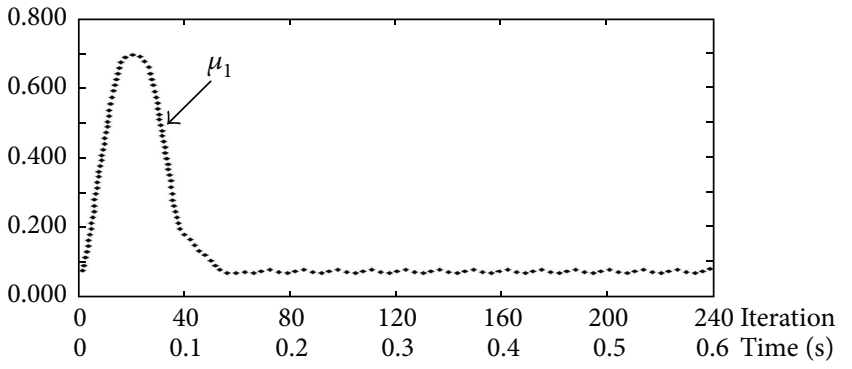

(a)

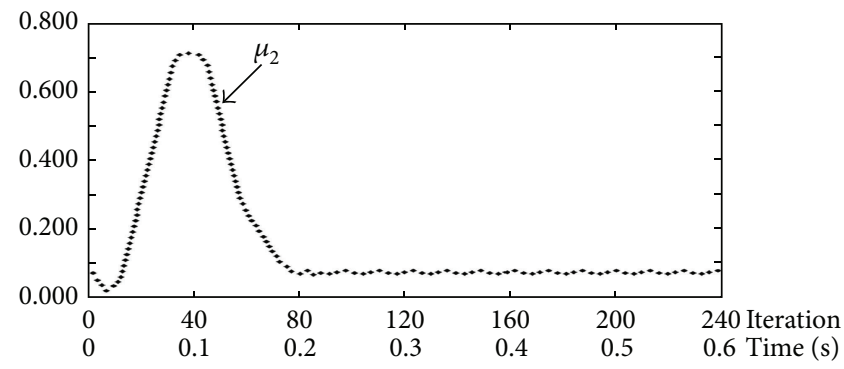

(b)

FiguRE 15: Numerical results of the iteration performance for two learning rates in the recurrent Laguerre-orthogonal-polynomial NN by means of modified PSO from $0 \mathrm{~s}$ to $0.6 \mathrm{~s}$ with 240 iterations (runs) at $376.8 \mathrm{rad} / \mathrm{s}(3600 \mathrm{rpm})$ case under the lumped nonlinear external disturbances with twice parameter variations $T_{l}=2 \Delta T_{p}+T_{\text {un }}$ : (a) response of learning rates $\mu_{1}$; (b) response of learning rates $\mu_{2}$.

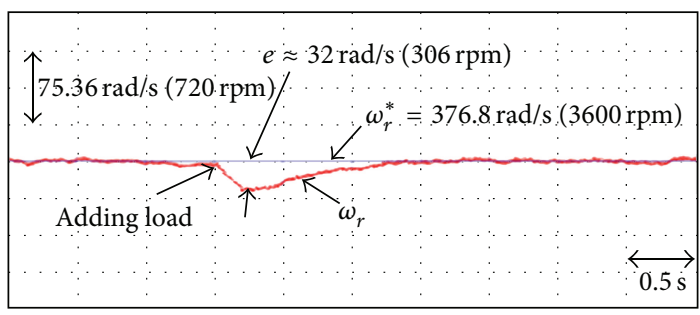

(a)

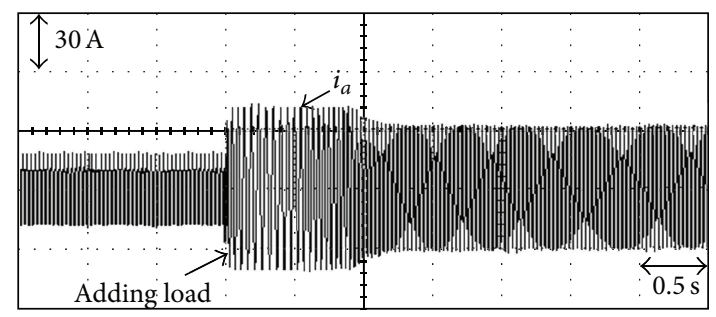

(b)

Figure 16: Experimental results under $T_{l}=2 N m\left(T_{a}\right)+T_{\text {un }}$ load disturbance with adding load at $376.8 \mathrm{rad} / \mathrm{s}(3600 \mathrm{rpm}) \mathrm{case}$ : (a) speed adjusted response of command rotor speed $\omega_{r}^{*}$ and measured rotor speed $\omega_{r}$ using the well-known PI controller; (b) response of measured current $i_{a}$ in phase $a$ using the well-known PI controller.

$2 \mathrm{Nm}\left(\mathrm{T}_{a}\right)+T_{\text {un }}$ load torque disturbance with adding load is tested by using the PI controller and the hybrid recurrent Laguerre-orthogonal-polynomial NN control system. The experimental result of load adjustment using wellknown PI controller under $T_{l}=2 \operatorname{Nm}\left(T_{a}\right)+T_{\text {un }}$ load torque disturbance with adding load at command rotor speed $376.8 \mathrm{rad} / \mathrm{s}(3600 \mathrm{rpm})$ is shown in Figure 16. The experimental results of the measured rotor speed response using well-known PI controller under $T_{l}=2 \mathrm{Nm}\left(T_{a}\right)+T_{\text {un }}$ load disturbance with adding load at $376.8 \mathrm{rad} / \mathrm{s}$ (3600 rpm) is shown in Figure 16(a). The experimental results of the measured current in phase $a$ using well-known PI controller under $T_{l}=2 \mathrm{Nm}\left(\mathrm{T}_{a}\right)+T_{\text {un }}$ load disturbance with adding load at $376.8 \mathrm{rad} / \mathrm{s}(3600 \mathrm{rpm})$ is shown in Figure 16(b). The experimental result of load adjustment using the hybrid recurrent Laguerre-orthogonal-polynomial NN control system under $T_{l}=2 \mathrm{Nm}\left(T_{a}\right)+T_{\text {un }}$ load torque disturbance with adding load at command rotor speed $376.8 \mathrm{rad} / \mathrm{s}$ (3600 rpm) is shown in Figure 17. The experimental results of the measured rotor speed response using the hybrid recurrent Laguerre-orthogonal-polynomial NN control system under $T_{l}=2 \mathrm{Nm}\left(T_{a}\right)+T_{\text {un }}$ load disturbance with adding load at $376.8 \mathrm{rad} / \mathrm{s}(3600 \mathrm{rpm})$ is shown in Figure $17(\mathrm{a})$. The experimental results of the measured current in phase $a$ 


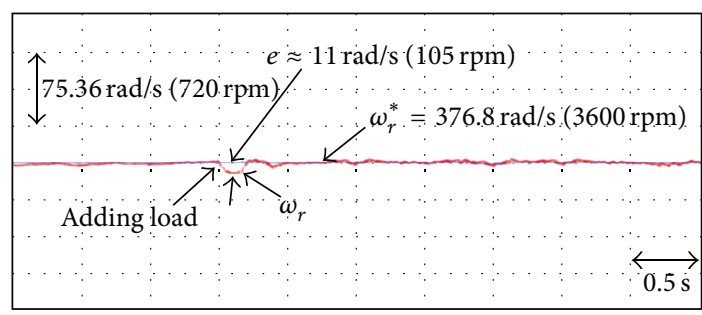

(a)

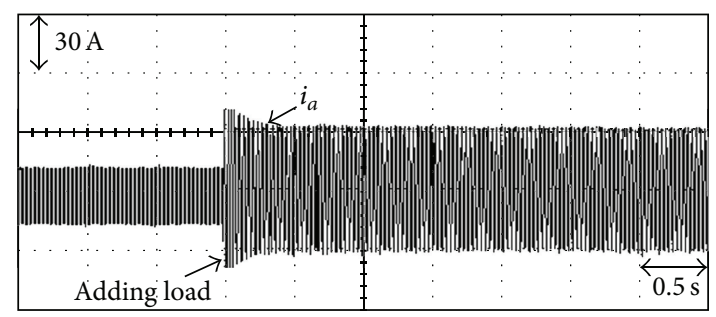

(b)

Figure 17: Experimental results under $T_{l}=2 N m\left(T_{a}\right)+T_{\text {un }}$ load disturbance with adding load at $376.8 \mathrm{rad} / \mathrm{s}(3600 \mathrm{rpm})$ case: (a) speed adjusted response of command rotor speed $\omega_{r}^{*}$ and measured rotor speed $\omega_{r}$ using the hybrid recurrent Laguerre-orthogonal-polynomial NN control system; (b) response of measured current $i_{a}$ in phase $a$ using the hybrid recurrent Laguerre-orthogonal-polynomial NN control system.

TABLE 1: Performances comparison of control systems.

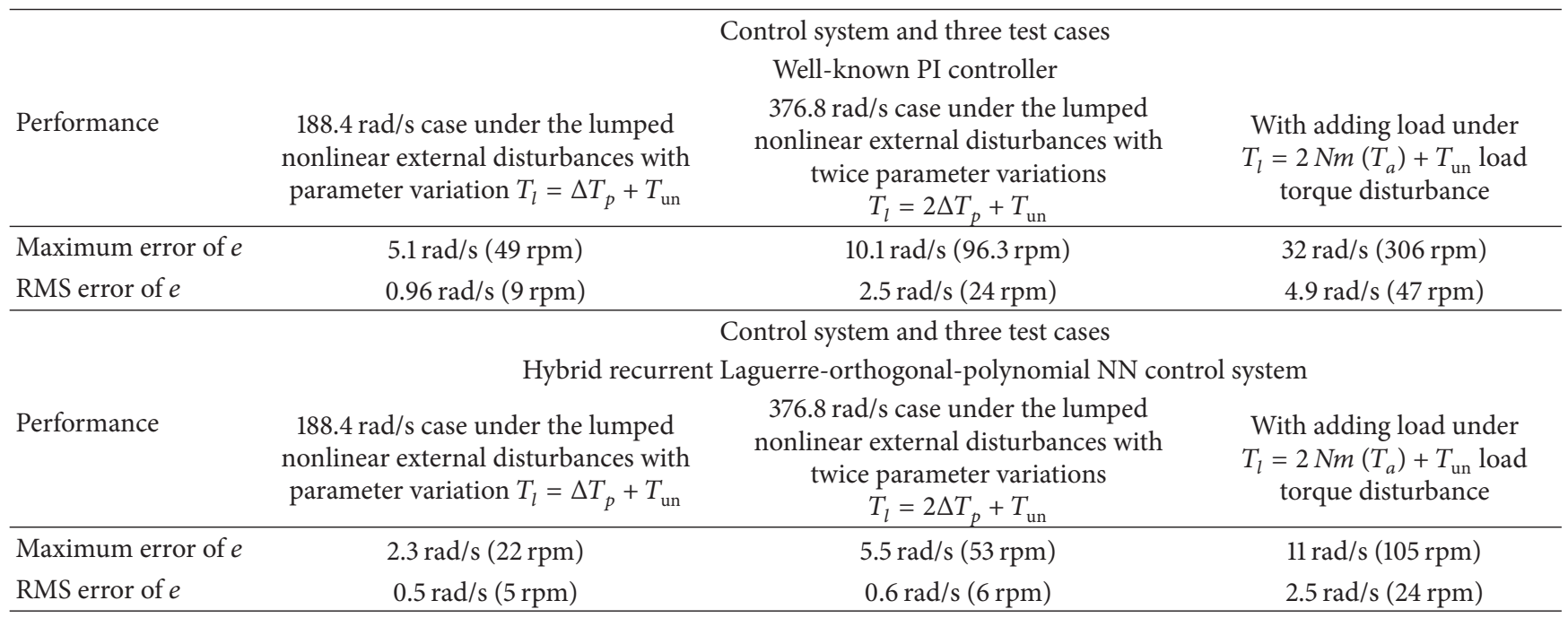

using the hybrid recurrent Laguerre-orthogonal-polynomial NN control system under $T_{l}=2 \mathrm{Nm}\left(T_{a}\right)+T_{\text {un }}$ load disturbance with adding load at $376.8 \mathrm{rad} / \mathrm{s}(3600 \mathrm{rpm})$ is shown in Figure 17(b). From the experimental results, the degenerated responses under $T_{l}=2 \mathrm{Nm}\left(T_{a}\right)+T_{\text {un }}$ load disturbance are much improved using the hybrid recurrent Laguerreorthogonal-polynomial NN control system. From experimental results, transient response of the hybrid recurrent Laguerre-orthogonal-polynomial NN control system has faster convergence and better load regulation than the wellknown PI controller at load regulation.

In addition, the control performance comparisons of the well-known PI controller and the hybrid recurrent Laguerreorthogonal-polynomial NN control system are summarized in Table 1 for experimental results at three test cases. In Table 1, the hybrid recurrent Laguerre-orthogonal-polynomial NN control system results in smaller tracking errors with respect to the well-known PI controller. According to the tabulated measurements, the proposed hybrid recurrent Laguerre-orthogonal-polynomial NN control system indeed yields the superior control performance compared to the well-known PI controller.

\section{Conclusions}

The hybrid recurrent Laguerre-orthogonal-polynomial NN control system has been successfully developed to control the V-belt CVT system driven by PMSM with robust control characteristics in this paper. The hybrid recurrent Laguerreorthogonal-polynomial NN control system with inspected control based on the uncertainty bounds of the controlled system was designed to stabilize the system states around a predetermined bound area. To drop the excessive and chattering resulted by control efforts, the hybrid recurrent Laguerre-orthogonal-polynomial NN control system, which is composed of the inspected control, the recurrent Laguerreorthogonal-polynomial NN control, and the recouped control, was proposed to reduce and smooth the control effort when the system states were inside the predetermined bound area.

The main contributions of this paper are as follows: (1) the dynamic models for the V-belt CVT driven by PMSM with unknown nonlinear and time-varying characteristics were successfully derived; (2) the hybrid recurrent Laguerreorthogonal-polynomial NN control system for the V-belt CVT system driven by PMSM under the occurrence of the 
lumped nonlinear load disturbances was successfully applied to enhance robustness; (3) the adaptive law of online parameters tuning in the recurrent Laguerre-orthogonal-polynomial $\mathrm{NN}$ and the estimation law of the recouped controller by using the Lyapunov stability theorem was successfully derived; (4) two optimal learning rates of connective weights and recurrent weights in the recurrent Laguerre-orthogonalpolynomial NN by means of modified PSO are successfully applied to speed up convergence; (5) the hybrid recurrent Laguerre-orthogonal-polynomial NN control system, which has better online learning capability in order to fast capture the system's nonlinear and time-varying behaviors, is successfully developed; (6) the proposed hybrid recurrent Laguerreorthogonal-polynomial NN control system has lower torque ripple than the well-known PI controller.

Finally, the control performance of the proposed hybrid recurrent Laguerre-orthogonal-polynomial NN control system is more suitable than the well-known PI controller for the V-belt CVT system driven by PMSM.

\section{Conflict of Interests}

The author declares that there is no conflict of interests regarding the publication of this paper.

\section{Acknowledgment}

The author would like to acknowledge the financial support of the Ministry of Science and Technology in Taiwan, through its Grant MOST 103-2221-E-239-016.

\section{References}

[1] C. Y. Tseng, L. W. Chen, Y. T. Lin, and J. Y. Li, "A hybrid dynamic simulation model for urban scooters with a mechanical-type CVT," in Proceedings of the IEEE International Conference on Automation and Logistics (ICAL '08), pp. 515-519, Qingdao, China, September 2008.

[2] C. Y. Tseng, Y. F. Lue, Y. T. Lin, J. C. Siao, C. H. Tsai, and L. M. $\mathrm{Fu}$, "Dynamic simulation model for hybrid electric scooters," in Proceedings of the IEEE International Symposium on Industrial Electronics (ISIE '09), pp. 1464-1469, Seoul, Republic of Korea, July 2009.

[3] L. Guzzella and A. M. Schmid, "Feedback linearization of sparkignition engines with continuously variable transmissions," IEEE Transactions on Control Systems Technology, vol. 3, no. 1, pp. 54-58, 1995.

[4] W. Kim and G. Vachtsevanos, "Fuzzy logic ratio control for a CVT hydraulic module," in Proceedings of the IEEE International Symposium on Intelligent Control, pp. 151-156, Rio, Greece.

[5] G. Carbone, L. Mangialardi, B. Bonsen, C. Tursi, and P. A. Veenhuizen, "CVT dynamics: theory and experiments," Mechanism and Machine Theory, vol. 42, no. 4, pp. 409-428, 2007.

[6] H. Sattler, "Efficiency of metal chain and V-belt CVT," in Proceedings of the International Conference on Continuously Variable Power Transmissions, pp. 99-104, Eindhoven, The Netherlands, 1999.

[7] G. Carbone, L. Mangialardi, and G. Mantriota, "The influence of pulley deformations on the shifting mechanism of metal belt
CVT,' Journal of Mechanical Design, vol. 127, no. 1, pp. 103-113, 2005.

[8] N. Srivastava and I. Haque, "A review on belt and chain continuously variable transmissions (CVT): dynamics and control," Mechanism and Machine Theory, vol. 44, no. 1, pp. 19-41, 2009.

[9] D. W. Novotny and T. A. Lipo, Vector Control and Dynamics of AC Drives, Oxford University Press, New York, NY, USA, 1996.

[10] R. Krishnan, Electric Motor Drives: Modeling, Analysis, and Control, Prentice Hall, Upper Saddle River, NJ, USA, 2001.

[11] F. J. Lin, "Real-time IP position controller design with torque feedforward control for pm synchronous motor," IEEE Transactions on Industrial Electronics, vol. 44, no. 3, pp. 398-407, 1997.

[12] K. S. Narendra and K. Parthasarathy, "Identification and control of dynamical systems using neural networks," IEEE Transactions on Neural Networks, vol. 1, no. 1, pp. 4-27, 1990.

[13] P. S. Sastry, G. Santharam, and K. P. Unnikrishnan, "Memory neuron networks for identification and control of dynamical systems," IEEE Transactions on Neural Networks, vol. 5, no. 2, pp. 306-319, 1994.

[14] R. Griñó, G. Cembrano, and C. Torras, "Nonlinear system identification using additive dynamic neural networks-two online approaches," IEEE Transactions on Circuits and Systems I, vol. 47, no. 2, pp. 150-165, 2000.

[15] Y. Li, "Stability of almost periodic solution for a general class of discontinuous neural networks with mixed time-varying delays," Mathematical Problems in Engineering, vol. 2013, Article ID 236189, 12 pages, 2013.

[16] W. Dong, X. Liu, and Y. Li, "Analysis of stiffened penstock external pressure stability based on immune algorithm and neural network," Mathematical Problems in Engineering, vol. 2014, Article ID 823653, 11 pages, 2014.

[17] Y. Pao, Adaptive Pattern Recognition and Neural Networks, Addison-Wesley, Boston, Mass, USA, 1989.

[18] Y. H. Pao and S. M. Phillips, "The functional link net and learning optimal control," Neurocomputing, vol. 9, no. 2, pp.149164,1995

[19] J. C. Patra, R. N. Pal, B. N. Chatterji, and G. Panda, "Identification of nonlinear dynamic systems using functional link artificial neural networks," IEEE Transactions on Systems, Man, and Cybernetics B: Cybernetics, vol. 29, no. 2, pp. 254-262, 1999.

[20] P. Aadaleesan, N. Miglan, R. Sharma, and P. Saha, "Nonlinear system identification using Wiener type Laguerre-Wavelet network model," Chemical Engineering Science, vol. 63, no. 15, pp. 3932-3941, 2008.

[21] S. Mahmoodi, J. Poshtan, M. R. Jahed-Motlagh, and A. Montazeri, "Nonlinear model predictive control of a $\mathrm{pH}$ neutralization process based on Wiener-Laguerre model," Chemical Engineering Journal, vol. 146, no. 3, pp. 328-337, 2009.

[22] A. Zou and X. Xiao, "An asynchronous encryption arithmetic based on Laguerre chaotic neural networks," in Proceedings of the WRI Global Congress on Intelligent Systems (GCIS '09), pp. 36-39, Xiamen, China, May 2009.

[23] J. C. Patra, C. Bornand, and P. K. Meher, "Laguerre neural network-based smart sensors for wireless sensor networks," in Proceeding of the IEEE Intrumentation and Measurement Technology Conference (I2MTC '09), pp. 832-837, Singapore, May 2009.

[24] J. C. Patra, P. K. Meher, and G. Chakraborty, "Development of laguerre neural-network-based intelligent sensors for wireless sensor networks," IEEE Transactions on Instrumentation and Measurement, vol. 60, no. 3, pp. 725-734, 2011. 
[25] T. W. S. Chow and Y. Fang, "A recurrent neural-network-based real-time learning control strategy applying to nonlinear systems with unknown dynamics," IEEE Transactions on Industrial Electronics, vol. 45, no. 1, pp. 151-161, 1998.

[26] M. A. Brdyś and G. J. Kulawski, "Dynamic neural controllers for induction motor," IEEE Transactions on Neural Networks, vol. 10, no. 2, pp. 340-355, 1999.

[27] X. D. Li, J. K. L. Ho, and T. W. S. Chow, "Approximation of dynamical time-variant systems by continuous-time recurrent neural networks," IEEE Transactions on Circuits and Systems II: Express Briefs, vol. 52, no. 10, pp. 656-660, 2005.

[28] C. H. Lu and C. C. Tsai, "Adaptive predictive control with recurrent neural network for industrial processes: an application to temperature control of a variable-frequency oil-cooling machine," IEEE Transactions on Industrial Electronics, vol. 55, no. 3, pp. 1366-1375, 2008.

[29] C. F. Hsu, "Intelligent position tracking control for LCM drive using stable online self-constructing recurrent neural network controller with bound architecture," Control Engineering Practice, vol. 17, no. 6, pp. 714-722, 2009.

[30] J. Kennedy and R. Eberhart, "Particle swarm optimization," in Proceedings of the IEEE International Conference on Neural Networks, vol. 4, pp. 1942-1948, Perth, Australia, December 1995.

[31] D. E. Goldberg, The Design of Innovation: Lessons from and for Competent Genetic Algorithms, Norwell, Kluwer Academic Publishers, Boston, Mass, USA, 2002.

[32] M. Clerc and J. Kennedy, "The particle swarm-explosion, stability, and convergence in a multidimensional complex space," IEEE Transactions on Evolutionary Computation, vol. 6, no. 1, pp. 58-73, 2002.

[33] A. B. de Carvalho, A. Pozo, and S. R. Vergilio, "A symbolic fault-prediction model based on multiobjective particle swarm optimization," Journal of Systems and Software, vol. 83, no. 5, pp. 868-882, 2010.

[34] Q. Li, W. Chen, Y. Wang, S. Liu, and J. Jia, "Parameter identification for PEM fuel-cell mechanism model based on effective informed adaptive particle swarm optimization," IEEE Transactions on Industrial Electronics, vol. 58, no. 6, pp. 24102419, 2011.

[35] C. T. Cheng, S. L. Liao, Z. T. Tang, and M. Y. Zhao, "Comparison of particle swarm optimization and dynamic programming for large scale hydro unit load dispatch," Energy Conversion and Management, vol. 50, no. 12, pp. 3007-3014, 2009.

[36] Y. X. Liao, J. H. She, and M. Wu, "Integrated hybrid-PSO and fuzzy-NN decoupling control for temperature of reheating furnace," IEEE Transactions on Industrial Electronics, vol. 56, no. 7, pp. 2704-2714, 2009.

[37] E. Zitzler, K. Deb, and L. Thiele, "Comparison of multiobjective evolutionary algorithms: empirical results," Evolutionary Computation, vol. 8, no. 2, pp. 173-195, 2000.

[38] R. C. Eberhart and Y. Shi, "Comparison between genetic algorithms and particle swarm optimization," in Proceedings of the 7th International Conference Evolutionary Programming, pp. 611-616, San Diego, Calif, USA, 1998.

[39] R. C. Eberhart and Y. Shi, "Comparing inertia weights and constriction factors in particle swarm optimization," in Proceedings of the Congress on Evolutionary Computation (CEC'00), pp. 8488, La Jolla, Calif, USA, July 2000.

[40] H. Gao and W. Xu, "A new particle swarm algorithm and its globally convergent modifications," IEEE Transactions on
Systems, Man, and Cybernetics B: Cybernetics, vol. 41, no. 5, pp. 1334-1351, 2011.

[41] T. Y. Sun, C. C. Liu, S. J. Tsai, S. T. Hsieh, and K. Y. Li, “Cluster guide particle swarm optimization (CGPSO) for underdetermined blind source separation with advanced conditions," IEEE Transactions on Evolutionary Computation, vol. 15, no. 6, pp. 798-811, 2011.

[42] Y. Zhang, X. Xiong, and Q. Zhang, "An improved selfadaptive PSO algorithm with detection function for multimodal function optimization problems," Mathematical Problems in Engineering, vol. 2013, Article ID 716952, 8 pages, 2013.

[43] C. H. Lin, P. H. Chiang, C. S. Tseng, Y. L. Liu, and M. Y. Lee, "Hybrid recurrent fuzzy neural network control for permanent magnet synchronous motor applied in electric scooter," in Proceeding of the International Power Electronics Conference, pp. 1371-1376, Sapporo, Japan, June 2010.

[44] C. H. Lin and C. P. Lin, "The hybrid RFNN control for a PMSM drive electric scooter using rotor flux estimator," International Journal of Electrical Power and Energy Systems, vol. 51, pp. 213223, 2013.

[45] C. H. Lin, "Hybrid recurrent wavelet neural network control of PMSM servo-drive system for electric scooter," International Journal of Control, Automation and Systems, vol. 12, no. 1, pp. 177-187, 2014.

[46] C. H. Lin, "Novel adaptive recurrent Legendre neural network control for PMSM servo-drive electric scooter," ASME-Journal of Dynamic Systems, Measurement and Control, vol. 137, no. 1, 12 pages, 2015.

[47] J. G. Ziegler and N. B. Nichols, "Optimum settings for automatic controllers," Transactions of the ASME, vol. 64, pp. 759-768, 1942.

[48] K. J. Astrom and T. Hagglund, PID Controller: Theory, Design, and Tuning, Instrument Society of America, Research Triangle Park, Raleigh, NC, USA, 1995.

[49] T. Hagglund and K. J. Astrom, "Revisiting the Ziegler-Nichols tuning rules for PI control," Asian Journal of Control, vol. 4, no. 4, pp. 364-380, 2002.

[50] T. Hagglund and K. J. Astrom, "Revisiting the Ziegler-Nichols tuning rules for PI control. Part II. The frequency response method," Asian Journal of Control, vol. 6, no. 4, pp. 469-482, 2004.

[51] J. J. E. Slotine and W. Li, Applied Nonlinear Control, Prentice Hall, Englewood Cliffs, NJ, USA, 1991.

[52] K. J. Astrom and B. Wittenmark, Adaptive Control, AddisonWesley, New York, NY, USA, 1995. 


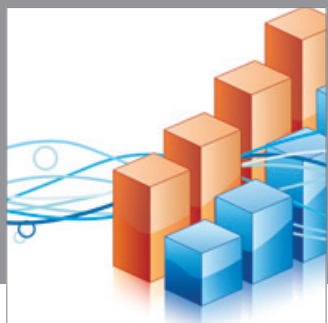

Advances in

Operations Research

mansans

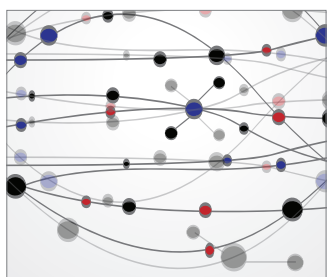

The Scientific World Journal
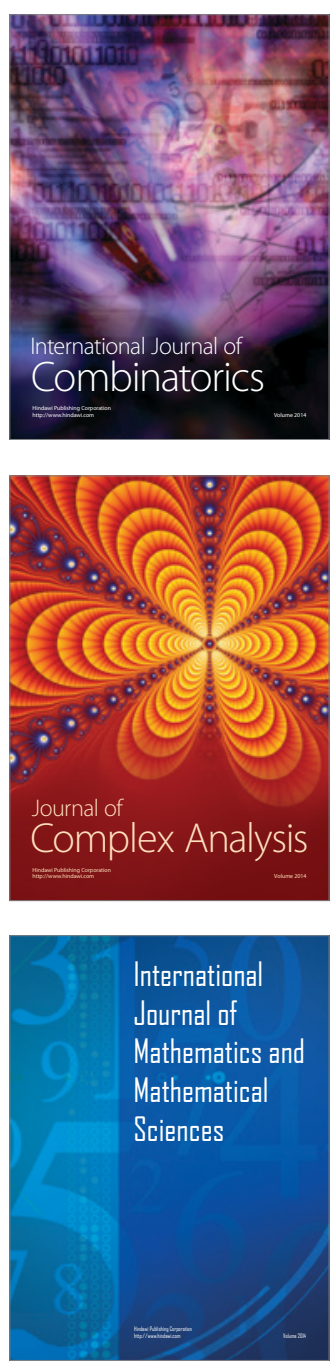
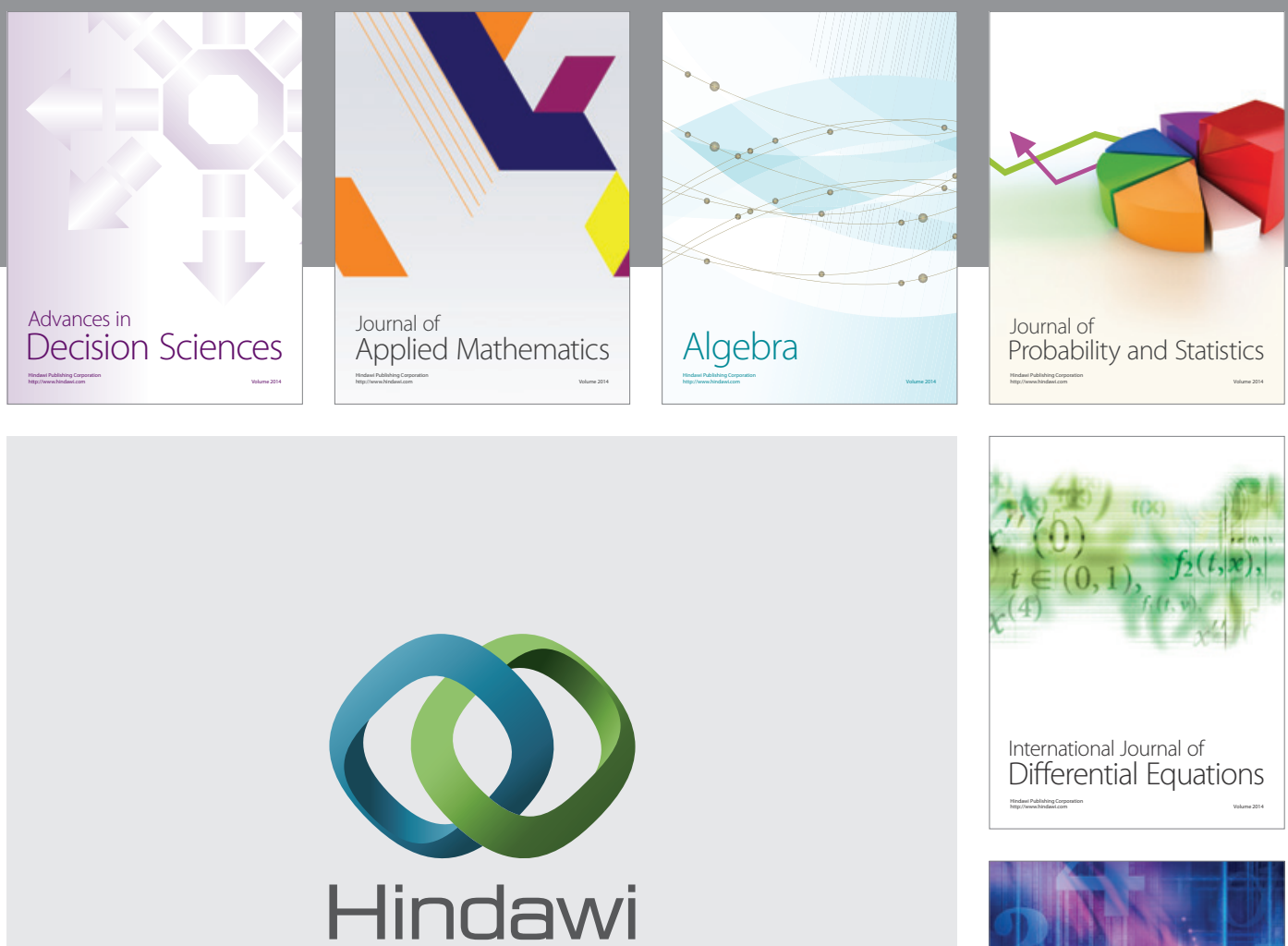

Submit your manuscripts at http://www.hindawi.com
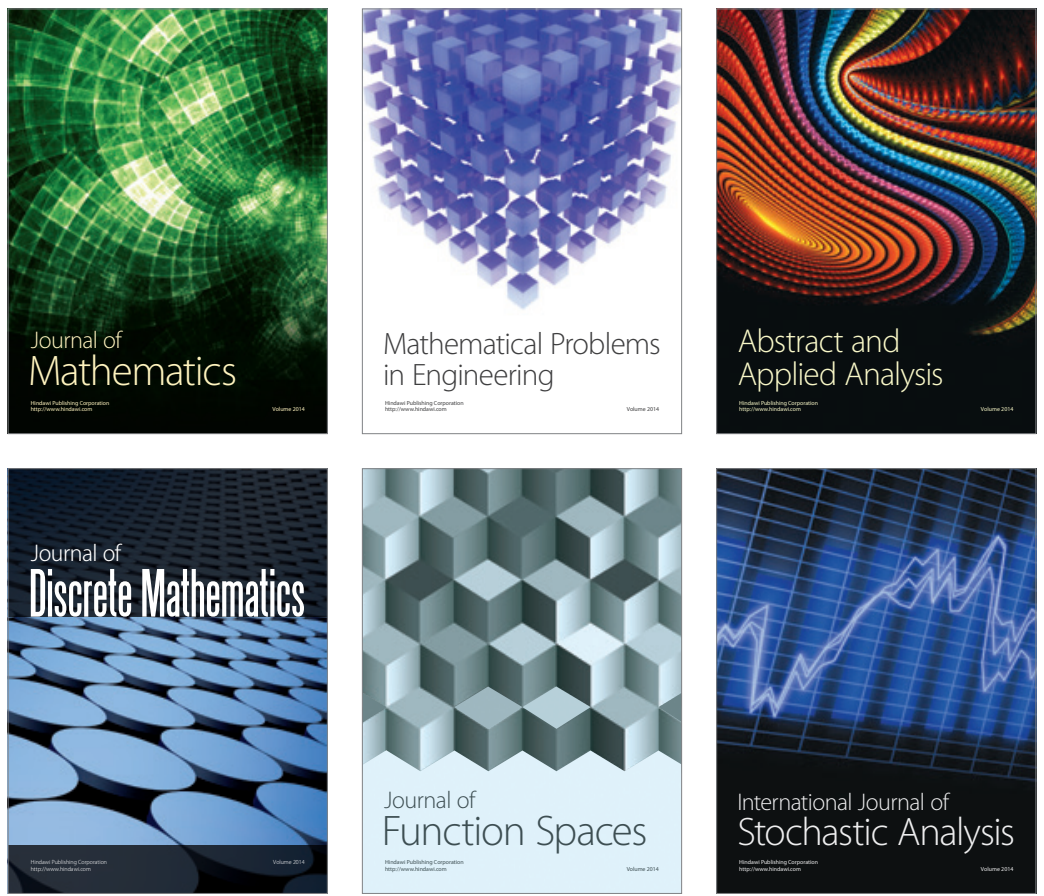

Journal of

Function Spaces

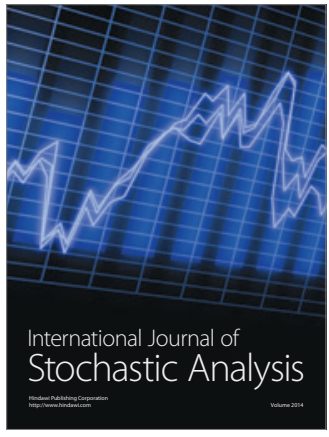

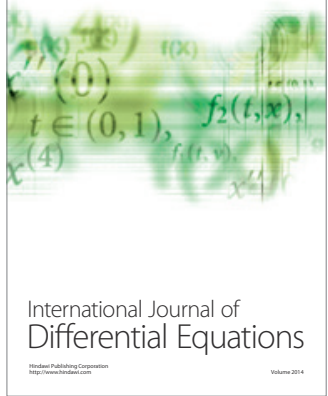
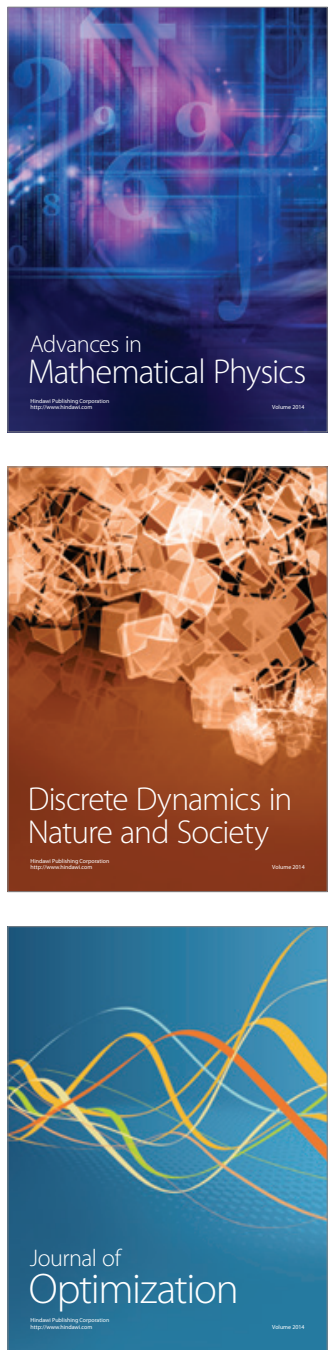